\title{
INIBIÇÃO DO CRESCIMENTO DE MICRORGANISMOS PATOGÊNICOS INDUZIDA POR BUTENOLÍDEOS ANÁLOGOS AOS CADIOLÍDEOS
}

\author{
Simone Z. Mairink ${ }^{\mathrm{a}}$, Luiz C. A. Barbosa ${ }^{\mathrm{a}, \mathrm{b}, *, ®}$, Célia R. A. Maltha ${ }^{\mathrm{a}}$, Jodieh O. S. Varejão $0^{\mathrm{a}, \mathrm{b}}$, Geane P. Oliveira ${ }^{\mathrm{b}}$, Jacqueline \\ A. Takahashi ${ }^{\mathrm{b}, *}$ e John Boukouvalas ${ }^{\mathrm{b}, \mathrm{c}, \text { (1) }}$ \\ ${ }^{a}$ Departamento de Química, Universidade Federal de Viçosa, Av. P. H. Rolfs s/n, 36570-900 Viçosa - MG, Brasil \\ 'Departamento de Química, Instituto de Ciências Exatas, Universidade Federal de Minas Gerais, Av. Pres. Antônio Carlos, 6627, \\ Campus Pampulha, CEP 31270-901, Belo Horizonte - MG, Brasil \\ 'Departamento de Química e Centro de Química Verde e Catálise, Pavillon Alexandre-Vachon, 1045 Avenue de la Médecine, \\ Université Laval, Quebec City, Quebec G1V 0A6, Canada
}

Recebido em 15/02/2019; aceito em 02/05/2019; publicado em 22/05/2019

\begin{abstract}
INHIBITORY EFFECT ON GROWTH OF PATHOGENIC MICROORGANISMS INDUCED BY BUTENOLIDES RELATED TO CADIOLIDES. The increasing number of antibiotic resistant microorganisms requires the development of new drugs. In this context, natural cadiolides are promising leads as they present potent antimicrobial activity and low cytotoxicity. In this work we report the synthesis of 21 new cadiolide analogues. The synthesis involved a Diels-Alder cycloaddition/ cycloreversion reaction between an oxazole and different ynones, affording four intermediate butenolides (14a-d) in 61-81\% yield. These intermediates were subjected to a vinylogous Knoevenagel condensation, followed by demethylation of the methoxylated compounds, affording all 21 analogues in good yields. The antibiotic activity of all compounds was evaluated against a panel of five microorganisms. The bioassays on the microbial growth showed that the cadiolides are more active for Gram-positive than Gram-negative bacteria, and have low activity against the fungus $C$. albicans. The most active compounds $(\mathbf{1 4 b}, \mathbf{1 5} \mathbf{b}, \mathbf{1 5} \mathbf{c}$ and $\mathbf{1 6 e})$ were effective against $S$. aureus showing $\mathrm{IC}_{50}$ in the range of 1.8-6.0 $\mu \mathrm{g} \mathrm{mL}^{-1}$. The results also indicated that the nature and position of substituent groups on the aromatic moieties have some effect on the activities.
\end{abstract}

Keywords: cadiolides; butenolides; growth inhibition; pathogenic microorganisms.

\section{INTRODUÇÃO}

Produtos naturais constituem uma importante fonte de novos compostos com potencial uso na descoberta de novos fármacos. ${ }^{1-2}$ Dentre as muitas fontes de produtos naturais disponíveis, existe um crescente interesse por compostos isolados de organismos marinhos. ${ }^{3}$ Isso se deve ao fato de que diversos metabólitos produzidos por espécies marinhas apresentam características estruturais únicas, com esqueletos carbônicos e atividades biológicas diversificadas, constituindo-se em modelos para o desenvolvimento de novas drogas. ${ }^{4-8}$

Dentre a grande variedade de metabólitos secundários de origem marinha encontram-se os cadiolídeos (Figura 1), que correspondem a butirolactonas contendo três anéis benzênicos. Geralmente os anéis benzênicos dos cadiolídeos apresentam um grupo $\mathrm{OH}$ ou $\mathrm{OCH}_{3}$, além de átomos de bromo em números variáveis. Os cadiolídeos A e B foram isolados de ascídias do gênero Botryllus, coletadas em Barrang Caddi na Indonésia. ${ }^{9}$ Os cadiolídeos B-I são produzidos por ascídias Pseudodistoma antinboja (cadiolídeos C-F) ${ }^{10}$ e do gênero Synoicum (cadiolídeos B, E, G-I). ${ }^{11,12}$

Os cadiolídeos B-F inibem cepas de Staphylococcus aureus resistentes a meticilina (SARM), com concentração inibitória mínima (MIC) na faixa de 0,3-2,0 $\mu \mathrm{g} \mathrm{mL}^{-1}$. Estes valores são comparáveis ou melhores do que aqueles apresentados por medicamentos como a Vancomicina $\left(\mathrm{MIC}=0,5-1,0 \mu \mathrm{g} \mathrm{mL}^{-1}\right)$, Linezolida $\left(2,0-4,0 \mu \mathrm{g} \mathrm{mL}^{-1}\right)$, Daptomicina $\left(<32 \mu \mathrm{g} \mathrm{mL}{ }^{-1}\right)$ e Platensimicina $\left(8,0 \mu \mathrm{g} \mathrm{mL}^{-1}\right) .{ }^{10} \mathrm{SARM}$ é uma séria ameaça para à saúde humana, não somente para pacientes hospitalizados, mas também para indivíduos saudáveis, podendo levar à morte. ${ }^{13}$

Além da atividade antibacteriana, foi relatado que o cadiolídeo

*e-mail: 1cab@ufmg.brou jat@qui.ufmg.br
E inibe fortemente a enzima isocitrato liase de Candida albicans, com $\mathrm{IC}_{50}$ de 7,62 $\mu \mathrm{M} .{ }^{14} \mathrm{O}$ antifúngico utilizado como controle positivo, 3-nitropropinato, foi menos ativo que o cadiolideo $\mathrm{E}$, com $\mathrm{IC}_{50}$ de $13,91 \mu \mathrm{M}$. Os cadiolídeos $\mathrm{H}$ e I também inibiram a isocitrato liase, apresentando $\mathrm{IC}_{50}$ de 17,16 e $10,36 \mu \mathrm{M}$, respectivamente. $\mathrm{O}$ cadiolídeo B também inibe, na concentração de $1 \mu \mathrm{g} \mathrm{mL}^{-1}$, o vírus Japanese encephalitis ${ }^{12}$.

Um aspecto importante sobre os cadiolídeos que são antimicrobianos é que todos os estudos realizados revelaram que esses compostos apresentam baixa atividade citotóxica. ${ }^{10,11-15}$

Em função das atividades listadas anteriormente, várias estratégias de síntese foram desenvolvidas para esses compostos (Esquema 1). ${ }^{15-18}$ A primeira síntese total de um cadiolídeo foi relatada em 2005 (Esquema 1, Estratégia 1). Neste trabalho, o cadiolídeo B foi sintetizado a partir da lactona 4-bromofuran-2 $(5 H)$-ona (1), gerando os intermediários 2 e 3, em $42 \%$ de rendimento global. ${ }^{15}$ Outra abordagem para obtenção do intermediário $\mathbf{3}$ foi relatada para a síntese dos cadiolídeos $\mathrm{A}, \mathrm{B}$ e $\mathrm{C}$, utilizando um processo multicomponente envolvendo o intermediário 4 como o passo proeminente (Esquema 1, Estratégia 2). ${ }^{16,17}$

Uma das metodologias mais elegantes para a síntese dos cadiolídeos A, B e D, utiliza como etapa-chave para a construção do núcleo $\beta$-aril- $\alpha$-benzoilbutenolídeo (2), foi uma reação de cicloadição/ cicloreversão de Diels-Alder entre a inona 5 e o oxazol $6{ }^{18}$ Esta etapa-chave ocorre em alto rendimento (70\%), e o intermediário 2 é submetido a uma reação de condensação de Knoevenagel viníloga com benzaldeídos (Esquema 1, Estratégia 3). Com essa metodologia os cadiolídeos A, B e D foram obtidos com rendimento global de 38 a 47\%. Apesar da elegância e eficiência dessa estratégia, ela ainda não foi explorada para a síntese de análogos aos cadiolídeos com vista à obtenção de novos agentes antimicrobianos. 
<smiles>[X]c1cc(/C=C2\OC(=O)C(C(=O)c3cc(Br)c(O)c(Br)c3)=C2c2cc([X])c(O)c(Br)c2)cc([X])c1[R20]</smiles>

Cadiolídeo A: $\mathrm{R}=\mathrm{X}=\mathrm{Z}=\mathrm{H} ; \mathrm{Y}=\mathrm{Br}$ B: $\mathrm{R}=\mathrm{H} ; \mathrm{X}=\mathrm{Y}=\mathrm{Z}=\mathrm{Br}$ C: $\mathrm{R}=\mathrm{X}=\mathrm{Y}=\mathrm{H} ; \mathrm{Z}=\mathrm{Br}$ D: $\mathrm{R}=\mathrm{X}=\mathrm{H} ; \mathrm{Y}=\mathrm{Z}=\mathrm{Br}$ E: $\mathrm{R}=\mathrm{Y}=\mathrm{H} ; \mathrm{X}=\mathrm{Z}=\mathrm{Br}$ F: $\mathrm{R}=\mathrm{CH}_{3} ; \mathrm{X}=\mathrm{Y}=\mathrm{H} ; \mathrm{Z}=\mathrm{Br}$<smiles>[R20]c1ccc(/C=C2\OC(=O)C([C@H](O)c3cc(Br)c(OC)c(Br)c3)=C2c2cc([Y])c(O)c(Br)c2)cc1[X]</smiles>

G: $\mathrm{R}=\mathrm{Y}=\mathrm{H} ; \mathrm{X}=\mathrm{Br}$

H: $\mathrm{R}=\mathrm{CH}_{3} ; \mathrm{X}=\mathrm{H} ; \mathrm{Y}=\mathrm{Br}$<smiles>COC(=O)C1=C(c2cc(Br)c(O)c(Br)c2)C(=O)OC1=C(c1cc(Br)c(O)c(Br)c1)c1cc(Br)c(O)c(Br)c1</smiles>

Figura 1. Fórmulas estruturais dos cadiolídeos naturais A-I.

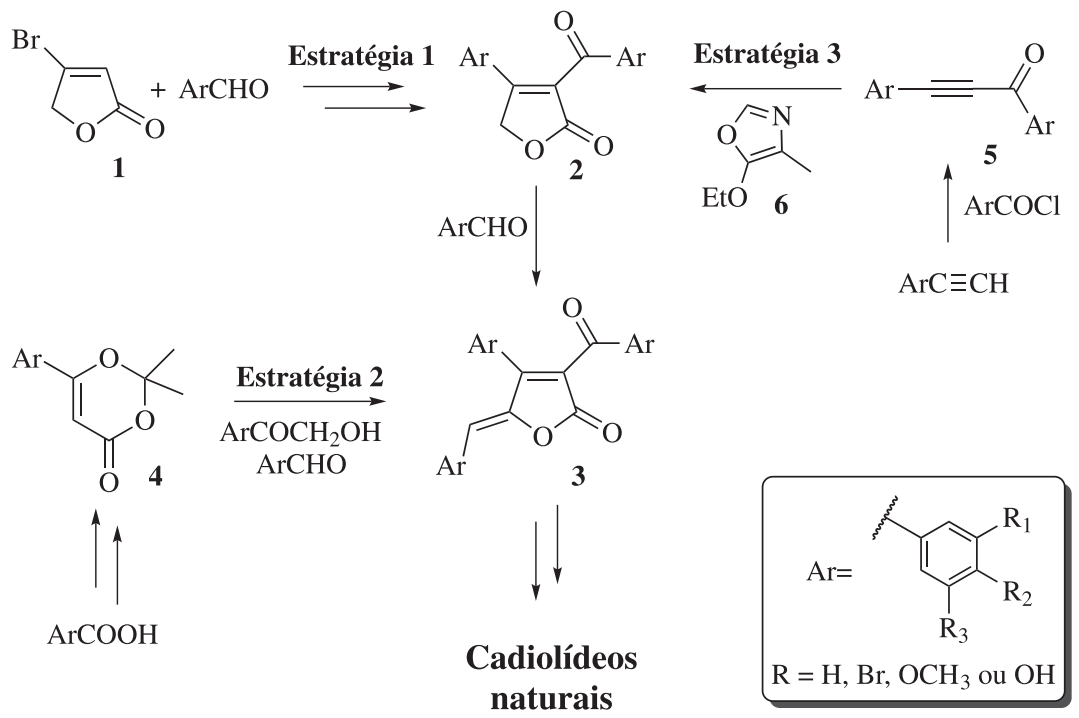

Esquema 1. Estratégias sintéticas para as sínteses totais de cadiolídeos naturais.

Nos últimos anos, nosso grupo tem relatado a síntese de vários butenolídeos análogos a compostos naturais e que apresentam atividades antibacteriana, ${ }^{19,20}$ herbicida ${ }^{21}$, inibidora de fotossíntese ${ }^{21-23} \mathrm{e}$ citotóxica. ${ }^{24}$ Dando continuidade a essa linha de pesquisa, e considerando que os cadiolídeos ainda foram pouco investigados em termos de atividade antimicrobiana, no presente artigo descrevemos a síntese de 21 novos análogos aos cadiolídeos empregando adaptações da metodologia desenvolvida por Boukouvalas e Thibault. ${ }^{18}$ Foram obtidos compostos com diferentes padrões de substituição nos três anéis benzênicos e a atividade antimicrobiana dos mesmos foi avaliada sobre os microrganismos patogênicos Candida albicans, Staphylococcus aureus, Bacillus cereus, Escherichia coli e Salmonella typhimurium.

\section{PARTE EXPERIMENTAL}

\section{Procedimentos gerais e equipamentos}

Todos os reagentes foram adquiridos da Sigma-Aldrich e foram utilizados sem qualquer purificação. Os solventes empregados nos procedimentos de cromatografia em coluna foram purificados conforme metodologias descritas na literatura. ${ }^{25}$ As reações foram monitoradas por cromatografia em camada delgada (CCD) utilizando-se placas de sílica gel Camlab-Polygram SILK/UV ${ }_{254}$, com 0,25 mm de espessura. As placas foram visualizadas em uma câmara de luz ultravioleta (254 e $365 \mathrm{~nm}$ ) ou reveladas com solução de ácido fosfomolíbdico ou permanganato de potássio. ${ }^{26}$ Os compostos foram isolados e purificados por cromatografia em coluna de sílica gel 60 (230-400 mesh-ASTM, Merck). Quando necessário, os produtos foram recristalizados empregando-se mistura de solventes.

As temperaturas de fusão foram determinadas em aparelho MQAPF-301 e não foram corrigidas. Os espectros no infravermelho (IV) de reflectância total atenuada foram obtidos em espectrofotômetro FT-IR VARIAN 660 equipado com GladiATR.

Os espectros de RMN foram adquiridos em um espectrômetro Varian Mercury-300, com frequência operacional de $300 \mathrm{MHz}$ para ${ }^{1} \mathrm{H}$ e $75 \mathrm{MHz}$ para ${ }^{13} \mathrm{C}$. Foi empregada uma sonda de $5 \mathrm{~mm}$ e detecção direta e unidade de controle de temperatura. Os espectros foram obtidos com as amostras dissolvidas em clorofórmio deuterado, dimetilsulfóxido deuterado ou outro solvente deuterado conforme especificado em cada caso, empregando-se tetrametilsilano como referência interna. Os espectros de massas foram obtidos em um espectrômetro Shimadzu QP5050A por inserção direta. Para os produtos finais inéditos os espectros de massas de alta resolução, no modo de ionização por electrospray, foram registrados em equipamento Finnigan MAT LCQ 7000. 


\section{Procedimentos sintéticos}

\section{3-(3-bromo-4-metoxifenil)-1-(3,5-dibromo-4-metoxifenil)prop-2- in-1-ona (11a)}

A uma solução de $\mathrm{PPh}_{3}(2,44 \mathrm{~g} ; 9,30 \mathrm{mmol})$ e $\mathrm{CBr}_{4}(1,54 \mathrm{~g}$ $4,65 \mathrm{mmol}$ ) em $5 \mathrm{~mL}$ de diclorometano anidro foi adicionado lentamente uma solução de 4-metoxibenzaldeído (318 mg; 2,33 mmol) em $5 \mathrm{~mL}$ de diclorometano anidro, sob atmosfera de nitrogênio, agitação magnética e a $0{ }^{\circ} \mathrm{C}$. A mistura foi aquecida até temperatura ambiente, permanecendo sob agitação por 4 horas. $\mathrm{O}$ solvente foi evaporado e o sólido obtido foi solubilizado em THF $(5 \mathrm{~mL})$ e resfriado à -78 ${ }^{\circ} \mathrm{C}$ sob atmosfera de nitrogênio. Uma solução de LDA previamente preparada pela adição de $n$-BuLi $(5,32 \mathrm{~mL} ; 1,9 \mathrm{~mol} \mathrm{~L}-1 ; 7,45 \mathrm{mmol})$ em $N, N$-diisopropilamina ( $1,05 \mathrm{~mL} ; 7,45 \mathrm{mmol})$ solubilizada em THF anidro $(5,5 \mathrm{~mL})$, foi adicionada lentamente a $-78{ }^{\circ} \mathrm{C}$, permanecendo nesta temperatura por $1 \mathrm{~h}$. Decorrido este período, foi adicionado p-metoxibenzaldeído (324 mg; 2,38 mmol) em THF (4 mL) e a temperatura foi gradualmente elevada até $25^{\circ} \mathrm{C}$. A mistura reacional foi mantida sob agitação magnética por três horas. Solução saturada de $\mathrm{NH}_{4} \mathrm{Cl}(30 \mathrm{~mL})$ foi adicionada ao material resultante e a fase aquosa foi extraída com acetato de etila $(4 \times 30 \mathrm{~mL})$. A fase orgânica foi secada com sulfato de magnésio anidro, filtrada e concentrada sob pressão reduzida em evaporador rotativo, fornecendo o álcool desejado como um óleo marrom. Esse óleo foi dissolvido em diclorometano anidro (10 mL) e adicionado $\mathrm{MnO}_{2}(1,80 \mathrm{~g} ; 18,58 \mathrm{mmol})$. A mistura resultante foi mantida sob agitação magnética a $25^{\circ} \mathrm{C}$ por $17 \mathrm{~h}$. Após esse período, a mistura reacional foi filtrada sob celite e o solvente foi removido sob pressão reduzida em evaporador rotatório. A purificação por cromatografia em coluna de sílica gel eluída com hexano/AcOEt 1:1 (v/v) forneceu o composto 11a, obtido como um sólido de cor amarelo palha (533 mg; $2,00 \mathrm{mmol}, 86 \%) . \mathrm{T}_{\mathrm{f}}=72,7-73,6^{\circ} \mathrm{C} . \mathrm{R}_{\mathrm{f}}=0,56$ (hexano: acetato de etila, $1: 1 \mathrm{v} / \mathrm{v})$. IV $\left(\mathrm{cm}^{-1}\right) \bar{v}_{\max }: 3197,2933,2840$, 2188, 2041, 1629, 1593, 1506, 1459, 1251, 1157, 1110, 1019, 830, 754, 684, 595, 507. RMN de ${ }^{1} \mathrm{H}\left(300 \mathrm{MHz}, \mathrm{CDCl}_{3}\right) \delta 3,84(\mathrm{~s}, 3 \mathrm{H}$, 7'), 3,88 (s, 3H, 7'”), 6,92 (d, $J_{3^{\prime},{ }^{\prime}}=J_{5,6}=8,4 \mathrm{~Hz}, 2 \mathrm{H}, 3$ ' e 5'), 6,97 $\left(\mathrm{d}, J_{3, ",{ }^{\prime}}=J_{5,, 6}=8,5 \mathrm{~Hz}, 2 \mathrm{H}, 3\right.$ " e $\left.5 "\right), 7,62\left(\mathrm{~d}, J_{2,3}, J_{6,5}=8,4 \mathrm{~Hz}\right.$, $2 \mathrm{H}, 2$ ' e 6'), 8,18 (d, $J_{2,, 3}=J_{6 ", 5}=8,5 \mathrm{~Hz}, 2 \mathrm{H}, 2$ " e 6"). RMN de ${ }^{13} \mathrm{C}\left(75 \mathrm{MHz}, \mathrm{CDCl}_{3}\right.$ ) $\delta 55,5$ (7'), 55,6 (7') ), 86,8 (2), 93,5 (1), 112,1 (1'), 113,8 (3" e 5"), 114,4 (3' e 5'), 130,4 (1"), 131,9 (2" e 6"), 135,0 (2' e 6'), 161,5 (4'), 164,3 (4’), 176,8 (3). EM, m/z (\%) 266 $\left(\left[\mathrm{M}^{+}\right], \mathrm{C}_{17} \mathrm{H}_{14} \mathrm{O}_{3}, 79\right), 267\left([\mathrm{M}+1]^{+}, 14\right), 239(12), 238$ (71), 224 (16), 223 (100), 195 (28), 180 (12), 163 (7), 160 (7), 159 (71), 152 (29), 151 (10), 144 (18), 135 (20), 131 (6), 126 (7), 119 (35), 116 (23), 107 (7), 92 (24), 88 (21), 87 (8), 77 (43), 76 (11), 75 (13), 74 (11), 64 (24), 63 (36), 62 (23), 51 (15), 50 (14).

\section{3-(3-bromo-4-metoxifenil)-1-(4-metoxifenil)prop-2-in-1-ona (11b)}

A uma solução de $\mathrm{Pd}\left(\mathrm{PPh}_{3}\right)_{2} \mathrm{Cl}_{2}(19 \mathrm{mg} ; 0,028 \mathrm{mmol})$ e $\mathrm{CuI}$ (11 mg; 0,056 mmol) em $5 \mathrm{~mL}$ de THF anidro mantida sob agitação magnética a $25^{\circ} \mathrm{C}$ por 30 minutos, sob atmosfera de nitrogênio, foram adicionados os seguintes reagentes: $\mathrm{Et}_{3} \mathrm{~N}(240 \mu \mathrm{L} ; 1,70 \mathrm{mmol})$, cloreto de 4-metoxibenzoíla (427 mg; 2,50 mmol) e 2-bromo-4-etinil-1-metoxibenzeno ( $347 \mathrm{mg} ; 1,70 \mathrm{mmol}$ ). A mistura resultante foi mantida sob agitação magnética por 3 horas. O solvente foi removido sob pressão reduzida e o material bruto foi purificado por cromatografia em coluna de sílica gel (hexano:éter dietílico 1:1 v/v) fornecendo o composto 11b como um sólido de cor amarelo palha $(458 \mathrm{mg}$; 1,33 mmol, 78\%). $\mathrm{T}_{\mathrm{f}}=136,8-137,2^{\circ} \mathrm{C} . \mathrm{R}_{\mathrm{f}}=0,25$ (hexano:éter dietílico, $1: 1 \mathrm{v} / \mathrm{v}) . \mathrm{IV}\left(\mathrm{cm}^{-1}\right) \bar{v}_{\max }: 3070,2962,2840,2194,2027,1629$, 1592, 1493, 1439, 1287, 1251, 1160,1017, 843, 806, 683, 597, 525. RMN de ${ }^{1} \mathrm{H}\left(300 \mathrm{MHz}, \mathrm{CDCl}_{3}\right.$ ) $\delta 3,89$ (s, 3H, 7'), 3,94 (s, 3H, 7'), $6,91\left(\mathrm{~d}, J_{5^{\prime}, 6^{\prime}}=8,6 \mathrm{~Hz}, 1 \mathrm{H}, 5^{\prime}\right), 6,98\left(\mathrm{~d}, J_{3^{\prime \prime}, 2^{\prime \prime}}=J_{5^{\prime \prime}, 6^{\prime \prime}}=8,9 \mathrm{~Hz}, 2 \mathrm{H}\right.$, 3 " e 5"), 7,61 (dd, $J_{6^{\prime}, 5}=8,6 \mathrm{~Hz}$ e $J_{6^{\prime}, 2},=2,0 \mathrm{~Hz}, 1 \mathrm{H}, 6$ '), 7,85 (d,
$\left.J_{2^{\prime}, 6^{\prime}}=2,0 \mathrm{~Hz}, 1 \mathrm{H}, 2^{\prime}\right), 8,16$ (d, $J_{2^{\prime \prime}, 3^{\prime \prime}}=J_{6^{\prime \prime}, 5^{\prime \prime}}=8,9 \mathrm{~Hz}, 2 \mathrm{H}, 2$ "' e 6"). $\mathrm{RMN} \mathrm{de}{ }^{13} \mathrm{C}\left(75 \mathrm{MHz}, \mathrm{CDCl}_{3}\right) \delta 55,6$ (7') $), 56,4$ (7'), 87,1 (2), 91,2 (1), 111,7 (5'), 111,8 (3'), 113,6 (1'), 113,9 (3"' e 5'), 130,2 (1'), 131,9 (2" e 6"), 134,0 (6'), 137,6 (2'), 157,9 (4'), 164,5 (4”), 176,4 (3). EM, $m / z(\%) 344\left(\left[\mathrm{M}^{+}\right], \mathrm{C}_{17} \mathrm{H}_{13} \mathrm{BrO}_{3}, 70\right), 345\left([\mathrm{M}+1]^{+}, 10\right), 346$ $\left([\mathrm{M}+2]^{+}, 66\right), 347\left([\mathrm{M}+3]^{+}, 11\right), 319(11), 318(63), 316(64), 304$ (14), 303 (90), 302 (15), 301 (93), 275 (15), 273 (15), 258 (10), 239 (32), 237 (34), 224 (11), 222 (21), 207 (13), 179 (13), 164 (18), 163 (29), 159 (32), 158 (29), 151 (35), 150 (27), 143 (20), 137 (12), 136 (10), 135 (47), 134 (10), 115 (13), 111 (12), 107 (21), 106 (13), 100 (15), 99 (14), 98 (14), 97 (10), 92 (57), 88 (14), 87 (60), 86 (20), 82 (11), 81 (22), 78 (10), 77 (100), 76 (25), 75 (37), 74 (28), 64 (58), 63 (76), 62 (32), 61 (15), 53 (20), 51 (28), 50 (35).

Os compostos 11c e 11d foram sintetizados utilizando o mesmo procedimento experimental descrito para o composto $\mathbf{1 1 b}$. Os dados físicos e espectroscópicos do composto 11c podem ser encontrados em Mairink et al. ${ }^{27}$

\section{3-(3-bromo-4-metoxifenil)-1-(3,5-dibromo-4-metoxifenil)prop-2- in-1-ona (11d)}

O composto 11d foi obtido como um sólido branco, em $85 \%$ de rendimento, após purificação por cromatografia em coluna de sílica gel. $\mathrm{T}_{\mathrm{f}}=167,1-167,7^{\circ} \mathrm{C} . \mathrm{R}_{\mathrm{f}}=0,25$ (hexano:diclorometano, 1:1 v/v). IV $\left(\mathrm{cm}^{-1}\right) \bar{v}_{\text {max }}:$ 3074, 2947, 2843, 2556, 2178, 1977, 1690, 1632, 1589, 1493, 1377, 1260, 1184, 1049, 977, 880, 815, 732, 688, 580, 512. RMN de ${ }^{1} \mathrm{H}\left(300 \mathrm{MHz}, \mathrm{CDCl}_{3}\right) \delta 3,96$ (s, 7H, 7' e 7"'), 6,94 (d, $\left.J_{5^{\prime}, 6^{\prime}}=8,5 \mathrm{~Hz}, 1 \mathrm{H}, 5^{\prime}\right), 7,64\left(\mathrm{dd}, J_{6^{\prime}, 5}=8,5 \mathrm{~Hz}\right.$ e $J_{6^{\prime}, 2^{\prime}}=1,8 \mathrm{~Hz}, 1 \mathrm{H}$, 6'), 7,87 (d, $J_{2,6}$ ' $=1,8 \mathrm{~Hz}, 1 \mathrm{H}, 2^{\prime}$ ), 8,30 (s, 2H, 2'" e 6"). RMN de ${ }^{13} \mathrm{C}\left(75 \mathrm{MHz}, \mathrm{CDCl}_{3}\right) \delta 56,5$ (7'), 60,9 (7') $), 86,4$ (2), 93,6 (1), 111,8 (5'), 112,0 (3'), 112,7 (1'), 118,7 (3" e 5"), 133,8 (2" e 6"), 134,4 (6'), 134,8 (1'), 137,9 (2'), 158,4 (4'), 158,8 (4’), 174,1 (3). EM, $\mathrm{m} / \mathrm{z}(\%) 500\left(\left[\mathrm{M}^{+}\right], \mathrm{C}_{17} \mathrm{H}_{11} \mathrm{Br}_{3} \mathrm{O}_{3}, 35\right), 501\left([\mathrm{M}+1]^{+}, 14\right), 502\left([\mathrm{M}+2]^{+}\right.$, 100), $503\left([\mathrm{M}+3]^{+}, 25\right), 504\left([\mathrm{M}+4]^{+}, 100\right), 505\left([\mathrm{M}+5]^{+}, 20\right), 506$ $\left([\mathrm{M}+6]^{+}, 34\right), 478(12), 477(6), 476(32), 475(7), 474(32), 472(11)$, 463 (21), 462 (11), 461 (63), 460 (13), 459 (65), 458 (7), 457 (21), 423 (6), 418 (5), $416(5), 465$ (9), 271 (5), 240 (8), 239 (75), 238 (14), 237 (82), 230 (7), 228 (7), 224 (13), 222 (13), 194 (5), 149 (5), 143 (14), 115 (12), 114 (5), 87 (17), 75 (7), 74 (9), 62 (6).

\section{3-(4-metoxibenzoil)-4-(4-metoxifenil)-furan-2(5H)-ona (14a)}

A um tubo de vidro com tampa rosqueada $(13 \times 100 \mathrm{~mm})$ foram adicionados o composto 11a (202 mg; 0,80 mmol), 5-etoxi-4-metiloxazol (6) (558 mg; 4,40 mmol) e xileno (4 mL). A mistura foi mantida sob atmosfera de nitrogênio, o tubo foi vedado e recoberto com papel alumínio. A mistura reacional foi mantida sob agitação magnética por 24 horas, a $145-150{ }^{\circ} \mathrm{C}$. Após resfriamento, os produtos voláteis foram removidos sob pressão reduzida a $60{ }^{\circ} \mathrm{C}$ em um evaporador rotativo. O material bruto foi dissolvido em THF $(10 \mathrm{~mL})$ e uma solução aquosa $48 \%$ de $\operatorname{HBr}(45 \mu \mathrm{L} ; 0,40 \mathrm{mmol})$ foi adicionada lentamente. A mistura reacional foi mantida sob agitação magnética por 8 horas, a $25^{\circ} \mathrm{C}$. Uma solução saturada de $\mathrm{NaCl}(20 \mathrm{~mL})$ foi adicionada e a fase aquosa foi extraída com acetato de etila $(2 \times 30 \mathrm{~mL})$. A fase orgânica foi secada com $\mathrm{MgSO}_{4}$ e concentrada em evaporador rotatório sob pressão reduzida. O óleo marrom escuro obtido foi purificado por cromatografia em coluna de sílica gel (hexano:acetato de etila; $1: 1 \mathrm{v} / \mathrm{v}$ ), resultando no composto 14a como um sólido alaranjado $(210 \mathrm{mg}$; 0,65 mmol, 81\%). $\mathrm{T}_{\mathrm{f}}=165,8-166,6^{\circ} \mathrm{C} . \mathrm{R}_{\mathrm{f}}=0,25$ (hexano: acetato de etila, $1: 1 \mathrm{v} / \mathrm{v})$. IV $\left(\mathrm{cm}^{-1}\right) \bar{v}_{\text {max }}: 3015,2930,2838,2045,1732,1654$, 1594, 1517, 1420, 1307, 1248, 1160, 1056, 1022, 919, 878, 829, 793, 725, 649, 556, 474. RMN de ${ }^{1} \mathrm{H}\left(300 \mathrm{MHz}, \mathrm{CDCl}_{3}\right) \delta 3,78$ (s, 3H, 7'), 3,83 (s, 3H, 7' '), 5,29 (s, 2H, 5), 6,83 (d, $J_{3^{\prime}, 2^{\prime}}=J_{5^{\prime}, 6^{\prime}}=8,7 \mathrm{~Hz}$, $2 \mathrm{H}, 3^{\prime}$ e $\left.5^{\prime}\right), 6,90$ (d, $J_{3^{\prime \prime}, 2,}=J_{5^{\prime \prime}, 6}$ " $=8,7 \mathrm{~Hz}, 2 \mathrm{H}, 3^{\prime \prime}$ e 5"), 7,34 (d, $J_{2^{\prime}, 3^{\prime}}=J_{6,5},=8,7 \mathrm{~Hz}, 2 \mathrm{H}, 2^{\prime}$ e 6'), 7,90 (d, $J_{2^{\prime \prime}, 3^{\prime \prime}}=J_{6^{\prime \prime}, 5^{\prime}}=8,7 \mathrm{~Hz}, 2 \mathrm{H}$, 
2" e 6"). RMN de ${ }^{13} \mathrm{C}$ (75 MHz, $\mathrm{CDCl}_{3}$ ) $\delta$ 55,5 (7'), 55,6 (7"), 70,4 (5), 114,2 (3"' e 5"), 114,7 (3' e 5'), 121,5 (1'), 123.4 (4), 128.7 (1'), 129.7 (2' e 6'), 132.1 (2” e 6"), 159.9 (3), 162,5 (4'), 164,7 (4"), 171,4 (2), 190,3 (6). EM, m/z (\%) $324\left(\left[\mathrm{M}^{+}\right], \mathrm{C}_{19} \mathrm{H}_{16} \mathrm{O}_{5}, 22\right)$, 325 ([M+1] $\left.]^{+}, 5\right), 323$ (23), 293 (17), 251 (6), 239 (6), 238 (7), 159 (10), 149 (13), 136 (9), 135 (100), 133 (25), 121 (24), 107 (21), 102 (6), 92 (32), 89 (8), 88 (8), 78 (9), 77 (69), 76 (12), 75 (6), 64 (34), 63 (23), 53 (7), 51 (12), 50 (10).

Os compostos 14b-d foram sintetizados utilizando-se o mesmo procedimento experimental descrito para o composto $\mathbf{1 4 a}$. Os dados físicos e espectroscópicos do composto $\mathbf{1 4 b}$ podem ser encontrados em Mairink et al. ${ }^{27}$

\section{4-(3-bromo-4-metoxifenil)-3-(4-metoxibenzoil)furan-2(5H)-ona (14c)}

O composto 14c foi obtido como um sólido amarelo, em $79 \%$ de rendimento, após purificação por cromatografia em coluna de sílica gel. $\mathrm{T}_{\mathrm{f}}=155,8-156,7^{\circ} \mathrm{C} . \mathrm{R}_{\mathrm{f}}=0,34$ (hexano: acetato de etila, $1: 1 \mathrm{v} / \mathrm{v}) . \mathrm{IV}\left(\mathrm{cm}^{-1}\right) \bar{v}_{\text {max }}: 3484,3057,2932,2843,2574,2029,1744$, 1650, 1592, 1499, 1422, 1332, 1247, 1164, 1015, 890, 844, 733, 680, 609, 576, 435. RMN de ${ }^{1} \mathrm{H}\left(300 \mathrm{MHz}, \mathrm{CDCl}_{3}\right) \delta 3,84$ (s, 3H, 7'), 3,86 (s, 3H, 7'), 5,27 (s, 2H, 5), 6,81 (d, $J_{5,6},=8,7 \mathrm{~Hz}, 1 \mathrm{H}, 5^{\prime}$ ), $6,90\left(\mathrm{~d}, J_{3^{\prime \prime}, 2^{\prime}}=J_{5^{\prime \prime}, 6^{\prime \prime}}=8,7 \mathrm{~Hz}, 2 \mathrm{H}, 3\right.$ " e 5"), 7,35 (dd, $J_{6^{\prime}, 5}=8,7 \mathrm{~Hz}$ e $\left.J_{6^{\prime}, 2}=1,9 \mathrm{~Hz}, 1 \mathrm{H}, 6^{\prime}\right), 7,56\left(\mathrm{~d}, J_{2^{\prime}, 6^{\prime}}=1,9 \mathrm{~Hz}, 1 \mathrm{H}, 2^{\prime}\right), 7,87$ (d, $J_{2,3},=J_{6 ", 5}=8,7 \mathrm{~Hz}, 2 \mathrm{H}, 2$ " e 6"). RMN de ${ }^{13} \mathrm{C}\left(75 \mathrm{MHz}, \mathrm{CDCl}_{3}\right) \delta$ 55,6 (7’'), 56,4 (7'), 70,4 (5), 112,1 (5'), 112,5 (3'), 114,3 (3"' е 5”), 122,7 (1'), 124,7 (4), 128,6 (1"), 129,1 (6'), 132,1 (2'” e 6"), 132,4 (2'), 158,5 (3 e 4'), 164,8 (4'”), 171,0 (2), 189,8 (6). EM, m/z (\%) $402\left(\left[\mathrm{M}^{+}\right], \mathrm{C}_{19} \mathrm{H}_{15} \mathrm{Br}_{2} \mathrm{O}_{5}, 7\right), 404\left([\mathrm{M}+2]^{+}, 4\right), 148$ (19), 147 (5), 136 (8), 135 (100), 133 (5), 107 (18), 101 (5), 92 (28), 90 (5), 87 (5), 79 (5), 78 (6), 77 (65), 76 (9), 75 (9), 74 (7), 64 (21), 63 (22), 62 (7), 53 (9), 51 (17), 50 (8).

4-(3-bromo-4-metoxifenil)-3-(3,5-dibromo-4-metoxibenzoil)furan2(5H)-ona (14d).

O composto $\mathbf{1 4 d}$ foi obtido como um sólido amarelo, em $61 \%$ de rendimento, após purificação por cromatografia em coluna de sílica gel. $\mathrm{T}_{\mathrm{f}}: 173,4-173,9^{\circ} \mathrm{C} . \mathrm{R}_{\mathrm{f}}=0,33$ (hexano: acetato de etila, $2: 1 \mathrm{v} / \mathrm{v}$ ). IV $\left(\mathrm{cm}^{-1}\right) \bar{v}_{\text {max }}:$ 3457, 3082, 2918, 2849, 2361, 2030, 1740, 1663, 1585, 1502, 1440, 1334, 1259, 1158, 1039, 958, 874,826, 733, 659, 579, 514. RMN de ${ }^{1} \mathrm{H}\left(300 \mathrm{MHz}, \mathrm{CDCl}_{3}\right) \delta 3,92$ (s, 3H, 7'), 3,94 (s, $\left.3 \mathrm{H}, 7^{\prime \prime}\right), 5,32$ (s, 2H, 5), 6,88 (d, $J_{5^{\prime \prime}, 6^{\prime \prime}}=8,7 \mathrm{~Hz}, 1 \mathrm{H}, 5^{\prime}$ ), 7,38 (dd, $J_{6,5},=8,7 \mathrm{~Hz}$ e $\left.J_{6^{\prime}, 2},=2,1 \mathrm{~Hz}, 1 \mathrm{H}, 6^{\prime}\right), 7,60\left(\mathrm{~d}, J_{2,}, 6^{\prime}=2,1 \mathrm{~Hz}, 1 \mathrm{H}, 2^{\prime}\right)$, 8,02 (s, 2H, 2" e 6"). RMN de ${ }^{13} \mathrm{C}\left(75 \mathrm{MHz}, \mathrm{CDCl}_{3}\right) \delta 56,5$ (7'), 60,9 (7'), 70,6 (5), 112,2 (5'), 112,8 (3'), 119,0 (3"' e 5"), 122,3 (1'), 123,3 (4), 129,1 (6'), 132,6 (2'), 133,6 (1'), 133,9 (2”' e 6”), 159,1 (4'), 159,2 (4"), 161,0 (3), 170,4 (2), 188,1 (6). EM, $m / z$ (\%) 558 $\left(\left[\mathrm{M}^{+}\right], \mathrm{C}_{19} \mathrm{H}_{13} \mathrm{Br}_{3} \mathrm{O}_{5}, 35\right), 559\left([\mathrm{M}+1]^{+}, 30\right), 560\left([\mathrm{M}+2]^{+}, 100\right), 561$ $\left([\mathrm{M}+3]^{+}, 43\right), 562\left([\mathrm{M}+4]^{+}, 96\right), 563\left([\mathrm{M}+5]^{+}, 28\right), 564\left([\mathrm{M}+6]^{+}, 33\right)$, $565\left([\mathrm{M}+7]^{+}, 8\right), 533(14), 532(10), 531(28), 529(25), 503(10), 483$ (23), 482 (10), 481 (46), 479 (25), 453 (11), 384 (15), 382 (15), 358 (11), 356 (11), 297 (11), 296 (27), 294 (53), 293 (30), 292 (89), 290 (47), 239 (19), 237 (19), 215 (13), 213 (26), 211 (15), 201 (12), 200 (24), 148 (60), 105 (10), 77 (17), 75 (16), 74 (9), 62 (9).

\section{(Z)-3-(4-metoxibenzoil)-5-(4-metoxibenzilideno)-4-(4-metoxifenil)} furan-2(5H)-ona (15a)

Uma solução da lactona 14a (50 mg; 0,154 mmol), 4-metoxibenzaldeído (24,5 mg; 0,18 mmol), TBDMSOTf (40 $\mu \mathrm{L} ; 0,175 \mathrm{mmol}) \mathrm{e}$ DIPEA ( $48 \mu \mathrm{L} ; 0,269 \mathrm{mmol}$ ) em $5 \mathrm{~mL}$ de diclorometano anidro foi mantida sob agitação magnética a $25^{\circ} \mathrm{C}$ por 1 hora, sob atmosfera de nitrogênio. A mistura reacional foi refluxada a $40{ }^{\circ} \mathrm{C}$ e foi adicionado DBU (40 $\mu \mathrm{L} ; 0,269 \mathrm{mmol})$, mantendo o refluxo por 2 horas.
A sistema foi resfriado e transferida para um funil de separação extração com $10 \mathrm{~mL}$ de diclorometano. A fase orgânica resultante foi lavada com solução aquosa de $\mathrm{HCl} 1 \mathrm{M}(5 \mathrm{~mL})$ e solução saturada de $\mathrm{NaCl}(5 \mathrm{~mL})$, secada com $\mathrm{MgSO}_{4}$ anidro, filtrada e concentrada sob pressão reduzida. $\mathrm{O}$ material bruto da reação obtido como um resíduo marrom foi purificado por cromatografia em coluna de sílica gel (hexano:acetato de etila, 2:1 v/v) fornecendo o composto $15 \mathbf{a}$ como um sólido alaranjado (60,6 mg; 0,137 mmol, 89\%). $\mathrm{T}_{\mathrm{f}}=189,1$ $189,7^{\circ} \mathrm{C} . \mathrm{R}_{\mathrm{f}}=0,25$ (hexano: acetato de etila, $\left.2: 1 \mathrm{v} / \mathrm{v}\right) . \mathrm{IV}\left(\mathrm{cm}^{-1}\right) \bar{v}_{\max }$ : 3486, 3053, 2929, 2849, 2560, 2361, 2039, 1747, 1651, 1594, 1507, $1374,1251,1164,1114,1025,970,834,734,620,529,430 . \mathrm{RMN}$ de ${ }^{1} \mathrm{H}\left(300 \mathrm{MHz}, \mathrm{CDCl}_{3}\right) \delta 3,80$ (s, 3H, 7'), 3,82 (s, 3H, 7'"), 3,85 (s, 3H, 7"'), 6,23 (s, 1H, 7), 6,79-6,98 (m, 6H, 3', 3", 3'”, 5', 5" e 5'"'), 7,36 (d, $J_{2,3},=J_{6,5}$, $=8,6 \mathrm{~Hz}, 2 \mathrm{H}, 2^{\prime}$ e 6 '), 7,81 (d, $J_{2,, 3^{\prime}}=$ $J_{6 ", 5}=J_{2, ", 3,}=J_{6, ", 5}=8,5 \mathrm{~Hz}, 4 \mathrm{H}, 2$ ", 2 "', 6 " e 6"'). RMN de ${ }^{13} \mathrm{C}$ (75 MHz, $\mathrm{CDCl}_{3}$ ) $\delta$ 55,4 (7'), 55,4 (7"), 55,5 (7"”), 113,8 (3" e 5"), 114,3 (3' e 5'), 114,5 (3"' e 5'"'), 117,0 (7), 121,4 (1'), 122,7 (5), 125,7 (1'”), 129,3 (1'”), 130,8 (2' e 6'), 132,1 (2" e 6"), 133,0 (2"' e 6"'), 145,8 (4), 157,0 (3), 161,0 (4"'), 161,3 (4'), 164,2 (4"'), 166,8 (2), 188,3 (6). EM, $m / z(\%) 442\left(\left[\mathrm{M}^{+}\right], \mathrm{C}_{27} \mathrm{H}_{22} \mathrm{O}_{6}, 57\right), 443\left([\mathrm{M}+1]^{+}\right.$, 17), 334 (5), 333 (5), 303 (7), 251 (15), 227 (6), 148 (26), 136 (9), 135 (100), 121 (9), 120 (11), 107 (16), 92 (9), 91 (6), 77 (20), 57 (6).

Os compostos 15b-k foram sintetizados utilizando o mesmo procedimento experimental descrito para o composto 15a. Os dados físicos e espectroscópicos dos compostos 15b-e podem ser encontrados em Mairink et al.. ${ }^{27}$

\section{(Z)-4-(3-bromo-4-metoxifenil)-3-(4-metoxibenzoil)-5-(4-metoxi-} benzilideno)furan-2(5H)-ona (15f)

O composto $\mathbf{1 5 f}$ foi obtido como um sólido amarelo, em $73 \%$ de rendimento, após purificação por cromatografia em coluna de sílica gel. $\mathrm{T}_{\mathrm{f}}=177,4-178,3^{\circ} \mathrm{C} . \mathrm{R}_{\mathrm{f}}=0,32$ (hexano: acetato de etila, $3: 1 \mathrm{v} / \mathrm{v}$ ). IV $\left(\mathrm{cm}^{-1}\right) \bar{v}_{\max }: 3493,3054,2934,2840,2563,2361,2032,1746$, 1641, 1595, 1494, 1423, 1253, 1162, 1021, 974, 874, 734, 609, 555, 438. RMN de ${ }^{1} \mathrm{H}\left(300 \mathrm{MHz}, \mathrm{CDCl}_{3}\right.$ ) $\delta 3,84$ (s, 3H, 7" ), 3,86 (s, 3H, 7'”'), 3,90 (s, 3H, 7'), 6,19 (s, 1H, 7), 6,83-6,96 (m, 5H, 5', 3', 5', 3"' e 5"'), 7,36 (dd, $J_{6,5}$, $=8,5 \mathrm{~Hz}$ e $J_{6,2},=1,4 \mathrm{~Hz}, 1 \mathrm{H}, 6$ '), 7,63 (d, $\left.J_{2^{\prime}, 6}=1,4 \mathrm{~Hz}, 1 \mathrm{H}, 2^{\prime}\right), 7,81\left(\mathrm{~d}, J_{2^{\prime \prime}, 3^{\prime \prime}}=J_{6,5^{\prime \prime}}=J_{2^{\prime \prime}, 3^{\prime \prime},}=J_{6, ", 5^{\prime \prime}}=8,4 \mathrm{~Hz}\right.$, $4 \mathrm{H}, 2$ ", 6", 2"' e 6"'). RMN de ${ }^{13} \mathrm{C}\left(75 \mathrm{MHz}^{\circ} \mathrm{CDCl}_{3}\right) \delta 55,4$ (7"), 55,6 (7’”), 56,4 (7'), 111,8 (5'), 112,0 (3'), 113,9 (3” e 5”), 114,5 (3"' e 5"'), 117,2 (7), 122,6 (1'), 123,4 (5), 125,6 (1"'), 129,2 (1"), 130,0 (6'), 132,1 (2'” e 6"'), 133,2 (2" e 6"), 133,8 (2'), 145,5 (4), 155,6 (3), 157,5 (4'), 161,2 (4’”'), 164,3 (4”), 166,5 (2), 187,8 (6). $\mathrm{EM}, m / z(\%) 520\left(\left[\mathrm{M}^{+}\right], \mathrm{C}_{27} \mathrm{H}_{21} \mathrm{BrO}_{6}, 48\right), 521\left([\mathrm{M}+1]^{+}, 15\right), 522$ $\left([\mathrm{M}+2]^{+}, 47\right), 523\left([\mathrm{M}+3]^{+}, 13\right), 441(8), 331(5), 329(6), 227(11)$, 148 (28), 136 (10), 135 (100), 120 (16), 107 (17), 92 (13), 91 (8), 77 (26). HRMS [ESI(+), IT-TOF]: calculado $\left[\mathrm{C}_{27} \mathrm{H}_{22} \mathrm{BrO}_{6}\right]^{+}$521,0594; encontrado 521,0590 .

\section{(Z)-5-(3-bromo-4-metoxibenzilideno)-4-(3-bromo-4-metoxife- nil)-3-(4-metoxibenzoil)furan-2(5H)-ona $(\mathbf{1 5 g})$}

O composto $\mathbf{1 5 g}$ foi obtido como um sólido amarelo, em $76 \%$ de rendimento, após purificação por cromatografia em coluna de sílica gel. $\mathrm{T}_{\mathrm{f}}=191,6-192,2^{\circ} \mathrm{C} . \mathrm{R}_{\mathrm{f}}=0,35$ (hexano: acetato de etila, $3: 1 \mathrm{v} / \mathrm{v}$ ). IV $\left(\mathrm{cm}^{-1}\right) \bar{v}_{\text {max }}: 3505,3057,2929,2842,2566,2030,1748,1646,1592$, 1493, 1459, 1360, 1255, 1162, 1052, 1015, 976, 863, 734, 673, 609, 576, 441. RMN de ${ }^{1} \mathrm{H}\left(300 \mathrm{MHz}, \mathrm{CDCl}_{3}\right) \delta 3,84$ (s, 3H, 7"), 3,90 (s, 3H, 7'), 3,95 (s, 3H, 7'”), 6,10 (s, 1H, 7), 6,80 - 7,00 (m, 4H, 5', 3', 5" e 5'"), 7,30 - 7,47 (m, 1H, 6'), 7,55 - 7,70 (m, 1H, 2'), 7,75 - 7,87 (m, 3H, 2", 6" e 6"'), 7,94-8,04 (m, 1H, 2"'). RMN de ${ }^{13} \mathrm{C}\left(75 \mathrm{MHz}, \mathrm{CDCl}_{3}\right) \delta 55,6$ (7”), 56,4 (7'” e 7'), 111,9 (5'”), 111,9 (5'), 112,2 (3"'), 112,2 (3'), 114,0 (3" e 5"), 115,2 (7), 122,3 (1'), 124,1 (4), 126,8 (1'”), 129,0 (1"), 130,0 (6'), 131,9 (6”"), 132,1 
(2" e 6"), 133,7 (2'), 135,9 (2'”), 146,3 (4), 155,3 (3), 157,1 (4"'), 157,6 (4'), 164,4 (4”), 166,2 (2), 187,6 (6). EM, m/z (\%) 598 ([M+'], $\left.\mathrm{C}_{27} \mathrm{H}_{20} \mathrm{Br}_{2} \mathrm{O}_{6}, 12\right), 599\left([\mathrm{M}+1]^{+}, 4\right), 600\left([\mathrm{M}+2]^{+}, 24\right), 601\left([\mathrm{M}+3]^{+}\right.$, 7), $602\left([\mathrm{M}+4]^{+}, 13\right), 603\left([\mathrm{M}+5]^{+}, 4\right), 522(3), 521(4), 520(3), 519$ (3), 307 (3), 305 (3), 228 (8), 226 (8), 148 (4), 136 (8), 135 (100), 119 (6), 107 (13), 92 (6), 77 (14). HRMS [ESI(+), IT-TOF]: calculado $\left[\mathrm{C}_{27} \mathrm{H}_{21} \mathrm{Br}_{2} \mathrm{O}_{6}\right]^{+}$598,9699; encontrado 598,9700.

(Z)-4-(3-bromo-4-metoxifenil)-5-(3,5-dibromo-4-metoxibenzilideno)3-(4-metoxibenzoil)furan-2(5H)-ona (15h)

O composto $\mathbf{1 5 h}$ foi obtido como um sólido amarelo, em $55 \%$ de rendimento, após purificação por cromatografia em coluna de sílica gel. $T_{\mathrm{f}}=103,6-104,4^{\circ} \mathrm{C} . \mathrm{R}_{\mathrm{f}}=0,40$ (hexano: acetato de etila, $3: 1 \mathrm{v} / \mathrm{v}$ ). IV $\left(\mathrm{cm}^{-1}\right) \bar{v}_{\text {max }}: 3058,2929,2842,2566,2036,1759,1650,1594,1495$, $1421,1367,1259,1164,1053,980,865,733,608,524$. RMN de ${ }^{1} \mathrm{H}$ (300 MHz, $\mathrm{CDCl}_{3}$ ) $\delta 3,85$ (s, 3H, 7'”), 3,90 (s, 3H, 7'), 3,92 (s, 3H, 7"'), 6,03 (s, 1H, 7), 6,83 - 6,95 (m, 3H, 5', 3" e 5"), 7,30 - 7,41 (m, 1H, 6'), 7,56-7,67 (m, 1H, 2'), 7,80 (d, $J_{2,3}, 3^{\prime \prime}=J_{6 ", 5},=8,6 \mathrm{~Hz}$, 2H, 2" e 6"), 7,96 (s, 2H, 2" e 6" "). RMN de ${ }^{13} \mathrm{C}\left(75 \mathrm{MHz}, \mathrm{CDCl}_{3}\right)$ $\delta$ 55,6 (7'”), 56,4 (7'), 60,9 (7'”'), 111,9 (5'), 112,3 (3'), 112,8 (7), 114,1 (3" e 5"), 118,7 (3"' e 5"'), 121,8 (1"), 125,4 (5), 128,8 (1'), 129,9 (6'), 131,2 (1"”), 132,1 (2"' e 6"), 133,7 (2'), 134,7 (2"' e 6"'), 147,8 (4), 154,8 (3), 155,1 (4'”'), 157,8 (4'), 164,6 (4”), 165,6 (2), 187,3 (6). EM, $m / z(\%) 676\left(\left[\mathrm{M}^{+}\right], \mathrm{C}_{27} \mathrm{H}_{19} \mathrm{Br}_{3} \mathrm{O}_{6}, 5\right), 677\left([\mathrm{M}+1]^{+}, 2\right)$, $678\left([\mathrm{M}+2]^{+}, 15\right), 679\left([\mathrm{M}+3]^{+}, 5\right), 680\left([\mathrm{M}+4]^{+}, 15\right), 681\left([\mathrm{M}+5]^{+}\right.$, 5), $682\left([\mathrm{M}+6]^{+}, 5\right), 599$ (4), 136 (9), 135 (100), 107(12), 92 (6), 77 (13). HRMS [ESI(+), IT-TOF]: calculado $\left[\mathrm{C}_{27} \mathrm{H}_{20} \mathrm{Br}_{3} \mathrm{O}_{6}\right]^{+} 676,8805$; encontrado 676,8810 .

\section{(Z)-4-(3-bromo-4-metoxifenil)-5-(2,5-dimetoxibenzilideno)-}

\section{-3-(4-metoxibenzoil)furan-2(5H)-ona (15i)}

O composto $\mathbf{1 5 i}$ foi obtido como um sólido laranja, em $83 \%$ de rendimento, após purificação por cromatografia em coluna de sílica gel. $\mathrm{T}_{\mathrm{f}}=177,3-177,9^{\circ} \mathrm{C} . \mathrm{R}_{\mathrm{f}}=0,38$ (hexano: acetato de etila, $2: 1 \mathrm{v} / \mathrm{v}$ ). IV $\left(\mathrm{cm}^{-1}\right) \bar{v}_{\text {max }}: 3496,3071,2938,2839,2035,1752,1646,1596,1492$, 1421, 1259, 1228, 1164, 1019, 957, 868, 794, 714, 610, 521, 443. RMN de ${ }^{1} \mathrm{H}\left(300 \mathrm{MHz}, \mathrm{CDCl}_{3}\right.$ ) $\delta 3,79$ (s, 3H, 7'"'), 3,84 (s, 6H, 7', e 8"'), 3,90 (s, 3H, 7'), 6,77 - 6,94 (m, 6H, 7, 5', 3", 5", 3'” e 4"'), 7,35 - 7,42 (m, 1H, 6'), 7,64 - 7,70 (m, 1H, 2'), 7,79 - 7,87 (m, 3H, 2", 6" e 6"”). RMN de ${ }^{13} \mathrm{C}\left(75 \mathrm{MHz}, \mathrm{CDCl}_{3}\right) \delta 55,6$ (7"), 55,9 (8",), 56,2 (7'”), 56,3 (7'), 110,9 (7), 111,8 (3'”'), 111,8 (5'), 112,0 (3'), 113,9 (3" e 5"), 115,8 (6"”), 117,9 (4"”), 122,2 (1'), 122,5 (1"), 124,0 (5), 129,1 (1'”), 130,1 (6'), 132,1 (2" e 6"), 134,0 (2'), 146,7 (4), 152,8 (2"”), 153,7 (5"”), 155,4 (3), 157,5 (4'), 164,4 (4”), 166,3 (2), 187,9 (6). EM, $m / z(\%) 550\left(\left[\mathrm{M}^{+}\right], \mathrm{C}_{28} \mathrm{H}_{23} \mathrm{BrO}_{7}, 25\right), 551\left([\mathrm{M}+1]^{+}, 8\right)$, $552\left([\mathrm{M}+2]^{+}, 25\right), 553\left([\mathrm{M}+3]^{+}, 7\right), 472(5), 178(6), 163(12), 136$ (10), 135 (100), 121 (5), 107 (14), 92 (7), 77 (15). HRMS [ESI(+), IT-TOF]: calculado $\left[\mathrm{C}_{28} \mathrm{H}_{24} \mathrm{BrO}_{7}\right]^{+}$551,0700; encontrado 551,0705.

\section{(Z)-5-(3-bromo-4-metoxibenzilideno)-4-(3-bromo-4-metoxife-} nil)-3-(3,5-dibromo-4-metoxiben-zoil)furan-2(5H)-ona (15j)

O composto $\mathbf{1 5 j}$ foi obtido como um sólido amarelo, em $53 \%$ de rendimento, após purificação por cromatografia em coluna de sílica gel. $\mathrm{T}_{\mathrm{f}}=163,1-163,8^{\circ} \mathrm{C} . \mathrm{R}_{\mathrm{f}}=0,17$ (hexano: diclorometano, $3: 5 \mathrm{v} / \mathrm{v}$ ). IV $\left(\mathrm{cm}^{-1}\right) \bar{v}_{\text {max }}: 3063,2924,2851,2550,2027,1755,1656,1592,1494$, 1379, 1259, 1182, 1052, 983, 889,812, 735, 662, 576, 441. RMN de ${ }^{1} \mathrm{H}\left(300 \mathrm{MHz}, \mathrm{CDCl}_{3}\right) \delta 3,89$ (s, 3H, 7'"), 3,93 (s, 3H, 7'), 3,96 (s, $3 \mathrm{H}, 7$ '”), 6,17 (s, 1H, 7), 6,93 (d, $J_{5,6},=8,5 \mathrm{~Hz}, 1 \mathrm{H}, 5$ '), 6,95 (d, $J_{5, ", 6},=8,6 \mathrm{~Hz}, 1 \mathrm{H}, 5$ '”), 7,33 (dd, $J_{6,5},=8,5 \mathrm{~Hz}$ e $J_{6,2},=1,7 \mathrm{~Hz}, 1 \mathrm{H}$, 6'), 7,56 (d, $J_{2}, 6=1,7 \mathrm{~Hz}, 1 \mathrm{H}, 2$ ') , 7,83 - 7,89 (m, 3H, 2", 6" e 6"'), $8,00\left(\mathrm{~d}, J_{2},{ }^{\prime \prime},{ }^{\prime \prime}=1,9 \mathrm{~Hz}, 1 \mathrm{H}, 2{ }^{\prime \prime}\right)$. RMN de ${ }^{13} \mathrm{C}\left(75 \mathrm{MHz}, \mathrm{CDCl}_{3}\right) \delta$ 56,5 (7' e 7"'), 60,8 (7'), 111,9 (5'), 112,0 (5'”), 112,4 (3'), 112,4 (3"'), 117,2 (7), 118,5 (3" e 5"), 121,7 (5), 121,9 (1'), 126,6 (1"'),
130,0 (6'), 132,3 (6"'), 133,9 (1", 2" e 6"), 134,0 (2'), 136,2 (2'”), 145,9 (4), 157,5 (3), 157,6 (4”), 157,8 (4”'), 158,5 (4'), 165,6 (2), 185,7 (6). EM, $m / z$ (\%) $754\left(\left[\mathrm{M}^{+}\right], \mathrm{C}_{27} \mathrm{H}_{18} \mathrm{Br}_{4} \mathrm{O}_{6}, 15\right), 755\left([\mathrm{M}+1]^{+}, 5\right)$, $756\left([\mathrm{M}+2]^{+}, 55\right), 757\left([\mathrm{M}+3]^{+}, 16\right), 758\left([\mathrm{M}+4]^{+}, 79\right), 759\left([\mathrm{M}+5]^{+}\right.$, 23), $760\left([\mathrm{M}+6]^{+}, 53\right), 761\left([\mathrm{M}+7]^{+}, 15\right), 762\left([\mathrm{M}+8]^{+}, 15\right), 679(9)$, 677 (9), 411 (13), 410 (5), 409 (24), 407 (13), 295 (49), 294 (10), 293 (100), 291 (52), 250 (10), 248 (5), 239 (16), 237 (19), 235 (10), 228 (59), 226(60), 215 (29), 213 (47), 211 (20), 200 (11), 199 (10), 198 (10), 119 (54), 103 (11), 89 (15), 77 (8), 76 (9), 75 (10), 63 (5), 57 (6). HRMS [ESI(+), IT-TOF]: calculado $\left[\mathrm{C}_{27} \mathrm{H}_{19} \mathrm{Br}_{4} \mathrm{O}_{6}\right]^{+} 754,7910$; encontrado 754,7905 .

(Z)-4-(3-bromo-4-metoxifenil)-3-(3,5-dibromo-4-metoxibenzoil)-5-(3,5-dibromo-4-metoxibenzilideno)furan-2(5H)-ona $(\mathbf{1 5 k})$

O composto 15k foi obtido como um sólido amarelo, em $48 \%$ de rendimento, após purificação por cromatografia em coluna de sílica gel. $\mathrm{T}_{\mathrm{f}}=213,8-214,4{ }^{\circ} \mathrm{C} . \mathrm{R}_{\mathrm{f}}=0,28$ (hexano: diclorometano, $3: 5 \mathrm{v} / \mathrm{v}) . \mathrm{IV}\left(\mathrm{cm}^{-1}\right) \bar{v}_{\max }: 3069,2926,2853,2536,2162,1969,1765$, 1668,1592, 1470, 1380, 1260, 1185, 1054, 983, 892,812, 734, 661, 613, 437. RMN de ${ }^{1} \mathrm{H}\left(300 \mathrm{MHz}, \mathrm{CDCl}_{3}\right.$ ) $\delta 3,90$ (s, 3H, 7"), 3,93 (s, 6H, 7' e 7'"'), 6,09 (s, 1H, 7), 6,94 (d, $J_{5,}, 6^{\prime}=8,5 \mathrm{~Hz}, 1 \mathrm{H}, 5$ ') , 7,33 (dd, $J_{6,5},=8,5$ e $\left.J_{6^{\prime}, 2^{\prime}}=2,1 \mathrm{~Hz}, 1 \mathrm{H}, 6^{\prime}\right), 7,57$ (d, $\left.J_{2,}, 6^{\prime}=2,1 \mathrm{~Hz}, 1 \mathrm{H}, 2^{\prime}\right)$, 7,88 (s, 2H, 2" e 6"), 7,97 (s, 2H, 2" e 6""). RMN de ${ }^{13} \mathrm{C}(75 \mathrm{MHz}$, $\mathrm{CDCl}_{3}$ ) $\delta 56,5$ (7'), 60,8 (7'”), 60,9 (7'”'), 112,0 (5'), 112,6 (3'), 114,5 (7), 118,6 (3" e 5"), 118,8 (3"' e 5"'), 121,4 (1'), 123,2 (5), 130,0 (6'), 131,0 (1"'), 133,7 (1"), 133,9 (2'), 133,9 (2" e 6"), 134,9 (2"' e 6"'), 147,5 (4), 155,5 (4"'), 157,0 (3), 158,1 (4'), 158,7 (4”), 165,0 (2), 185,4 (6). EM, $m / z(\%) 832\left(\left[\mathrm{M}^{+}\right], \mathrm{C}_{27} \mathrm{H}_{17} \mathrm{Br}_{5} \mathrm{O}_{6}, 4\right), 834\left([\mathrm{M}+2]^{+}\right.$, 17), $835\left([\mathrm{M}+3]^{+}, 5\right), 836\left([\mathrm{M}+4]^{+}, 33\right), 837\left([\mathrm{M}+5]^{+}, 9\right), 838\left([\mathrm{M}+6]^{+}\right.$, $31), 839\left([\mathrm{M}+7]^{+}, 9\right), 840\left([\mathrm{M}+8]^{+}, 16\right), 841\left([\mathrm{M}+9]^{+}, 4\right), 757(5)$, 504 (5), 502 (5), 489 (7), 487 (6), 308 (13), 306 (26), 304 (14), 295 (44), 294 (9), 293 (100), 292 (7), 291 (68), 289 (12), 265 (5), 250 (7), 239 (12), 127 (16), 235 (9), 233 (5), 222 (5), 199 (5), 197 (5), 103 (9), 87 (6). HRMS [ESI(+), IT-TOF]: calculado $\left[\mathrm{C}_{27} \mathrm{H}_{18} \mathrm{Br}_{5} \mathrm{O}_{6}\right]^{+}$ 832,7015 ; encontrado 832,7020.

\section{(Z)-3-(4-hidroxibenzoil)-5-(4-hidroxibenzilideno)-4-(4-hidroxi- fenil)furan-2(5H)-ona (16a)}

A uma solução de $\mathbf{1 5 a}(50 \mathrm{mg} ; 0,113 \mathrm{mmol})$ em $3 \mathrm{~mL}$ de diclorometano anidro, resfriada a $-78^{\circ} \mathrm{C}$, sob atmosfera de nitrogênio e agitação magnética, foi adicionado uma solução de $1 \mathrm{~mol} \mathrm{~L}^{-1}$ de $\mathrm{BBr}_{3}$ em diclorometano (1,02 mL; 1,02 mol). Após a mistura alcançar à temperatura ambiente, manteve-se a agitação magnética por 20 horas. A mistura foi transferida para um funil de separação empregando-se $10 \mathrm{~mL}$ de água e extraiu-se com acetato de etila $(4 \times 10 \mathrm{~mL})$. A fase orgânica resultante foi lavada com solução saturada de $\mathrm{NaCl}$ (20 mL), secada com $\mathrm{MgSO}_{4}$ anidro e concentrada sob pressão reduzida para obtenção do material bruto da reação, que foi purificado por cromatografia em coluna de sílica gel empregando-se a mistura de solvente hexano:acetato de etila $1: 3 \mathrm{v} / \mathrm{v}$ para obtenção de $\mathbf{1 6 a}(37,1 \mathrm{mg}$; 0,093 mmol, 82\%). $\mathrm{T}_{\mathrm{f}}=245,8-246,4{ }^{\circ} \mathrm{C} . \mathrm{R}_{\mathrm{f}}=0,28$ (hexano: acetato de etila, $1: 3 \mathrm{v} / \mathrm{v})$. IV $\left(\mathrm{cm}^{-1}\right) \bar{v}_{\max }: 3270,2921,2851,2160,2020,1732$, 1595, 1501, 1376, 1275, 1160, 990, 879, 836, 685, 626, 531, 495. RMN de ${ }^{1} \mathrm{H}\left(300 \mathrm{MHz}, \mathrm{DMSO}-\mathrm{d}_{6}\right) \delta 6,32(\mathrm{~s}, 1 \mathrm{H}, 7), 6,65-6,94$ (m, 6H, 3', 3", 3'", 5', 5" e 5'"), 7,22 (d, $J_{2,3},=J_{6,5}=8,3 \mathrm{~Hz}, 2 \mathrm{H}, 2$ ' e 6'), 7,49 - 7,83 (m, 4H, 2", 2"', 6" e 6"'), 10,05 (s, 1H, 7'), 10,18 (s, 1H, 7"'), 10,57 (s, 1H, 7'"'). RMN de ${ }^{13} \mathrm{C}(75 \mathrm{MHz}$, DMSO-d 6 ) $\delta$ 116,0 (3" e 5"), 116,2 (3' e 5'), 116,5 (3"' e 5"'), 117,0 (7), 119,9 (1'), 121,8 (5), 124,5 (1'”), 127,9 (1'”), 131,3 (2" e 6"), 132,7 (2' e 6'), 133,5 (2"' e 6"'), 145,1 (4), 156,4 (3), 159,8 (4'), 160,0 (4"'), 163,5 (4”), 166,6 (2), 188,5 (6). EM, $m / z$ (\%) $400\left(\left[\mathrm{M}^{+} \cdot\right], \mathrm{C}_{24} \mathrm{H}_{16} \mathrm{O}_{6}\right.$, 84), $401\left([\mathrm{M}+1]^{+}, 25\right), 383$ (7), 306 (5), 305 (5), 280 (6), 252 (5), 239 (7), 223 (16), 199 (7), 145 (5), 135 (6), 134 (42), 122 (9), 121 
(100), 107 (7), 106 (13), 93 (21), 65 (12). HRMS [ESI(+), IT-TOF]: calculado $\left[\mathrm{C}_{24} \mathrm{H}_{17} \mathrm{O}_{6}\right]^{+}$401,1020; encontrado 401,1018.

Os compostos 16b-i foram sintetizados utilizando o mesmo procedimento experimental descrito para o composto 16a. Os dados físicos e espectroscópicos dos compostos $\mathbf{1 6 b}$-e podem ser encontrados em Mairink et al.. ${ }^{27}$

(Z)-4-(3-bromo-4-hidroxifenil)-3-(4-hidroxibenzoil)-5-(4-hidroxibenzilideno) furan-2(5H)-ona (16f)

O composto 16 foi obtido como um sólido amarelo, em $64 \%$ de rendimento, após purificação por cromatografia em coluna de sílica gel. $\mathrm{T}_{\mathrm{f}}=250,0-251,0{ }^{\circ} \mathrm{C}(\mathrm{dec}) . \mathrm{R}_{\mathrm{f}}=0,33$ (hexano: acetato de etila, $1: 3 \mathrm{v} / \mathrm{v})$. IV ( $\left.\mathrm{cm}^{-1}\right) \bar{v}_{\max }: 3300,3068,2922,2852,2162,1975,1731$, 1636, 1600, 1563, 1512, 1372, 1281, 1168, 984, 878, 826, 655, 609, 646. RMN de ${ }^{1} \mathrm{H}\left(300 \mathrm{MHz}, \mathrm{DMSO}_{6}\right) \delta$ 6,30 (s, 1H, 7), 6,76 (d, $J_{3^{\prime \prime}, 2,}=J_{5^{\prime \prime}, 6^{\prime \prime}}=8,6 \mathrm{~Hz}, 2 \mathrm{H}, 3$ " e 5"), 6,86 (d, $J_{3^{\prime \prime}, 2^{\prime \prime}}=J_{5, ", 6},=8,5 \mathrm{~Hz}$, $2 \mathrm{H}, 3$ " " e 5"'), 6,97 (d, $J_{5^{\prime}, 6}=8,4 \mathrm{~Hz}, 1 \mathrm{H}, 5$ '), 7,24 (dd, $J_{6,5},=8,4 \mathrm{~Hz}$ e $\left.J_{6^{\prime}, 2^{\prime}}=1,4 \mathrm{~Hz}, 1 \mathrm{H}, 6^{\prime}\right), 7,48$ (d, $\left.J_{2^{\prime}, 6^{\prime}}=1,4 \mathrm{~Hz}, 1 \mathrm{H}, 2^{\prime}\right), 7,60-7,84$ (m, 4H, 2", 6", 2"” e 6"'), 10,22 (s, 1H, 7"), 10,60 (s, 1H, 7"'), 10,92 (s, 1H, 7'). RMN de ${ }^{13} \mathrm{C}\left(75 \mathrm{MHz}, \mathrm{DMSO}-\mathrm{d}_{6}\right) \delta 110,0$ (3'), 115,9 (3" e 5"), 116,5 (3"' e 5"'), 116,9 (7), 117,1 (5'), 121,3 (1'), 122,6 (5), 124,5 (1'”), 127,9 (1"), 130,4 (6'), 132,7 (2"' e 6"'), 133,6 (2" e 6"), 133,9 (2'), 144,9 (4), 155,2 (3), 156,5 (4'), 159,9 (4'”'), 163,6 (4”), 166,4 (2), 188,1 (6). EM, m/z (\%) 478 ([M+'], $\left.\mathrm{C}_{24} \mathrm{H}_{15} \mathrm{BrO}_{6}, 30\right)$, $479\left([\mathrm{M}+1]^{+}, 9\right), 480\left([\mathrm{M}+2]^{+}, 30\right), 481\left([\mathrm{M}+3]^{+}, 8\right), 400(16), 360$ (5), 358 (5), 223 (7), 199 (7), 134 (37), 122 (8), 121 (100), 106 (13), 93 (19), 65 (10). HRMS [ESI(+), IT-TOF]: calculado $\left[\mathrm{C}_{24} \mathrm{H}_{16} \mathrm{BrO}_{6}\right]^{+}$ 479,0125; encontrado 479,0129.

(Z)-5-(3-bromo-4-hidroxibenzilideno)-4-(3-bromo-4-hidroxifenil)3-(4-hidroxibenzoil)furan-2(5H)-ona (16g)

O composto $\mathbf{1 6 g}$ foi obtido como um sólido laranja, em $71 \%$ de rendimento, após purificação por cromatografia em coluna de sílica gel. $\mathrm{T}_{\mathrm{f}}=151,8-151,5^{\circ} \mathrm{C} \cdot \mathrm{R}_{\mathrm{f}}=0,39$ (hexano: acetato de etila, $2: 1 \mathrm{v} / \mathrm{v}$ ). IV $\left(\mathrm{cm}^{-1}\right) \bar{v}_{\text {max }}: 3195,2921,1977,1728,1636,1565,1493,1367,1287$, 1160, 1043, 867, 818, 655, 609, 567, 424. RMN de ${ }^{1} \mathrm{H}(300 \mathrm{MHz}$, DMSO-d $)_{6} \delta 6,34(\mathrm{~s}, 1 \mathrm{H}, 7), 6,76\left(\mathrm{~d}, J_{3^{\prime \prime}, 2^{\prime \prime}}=J_{5^{\prime \prime}, 6^{\prime \prime}}=8,6 \mathrm{~Hz}, 2 \mathrm{H}, 3\right.$ " e 5'), 6,97 (d, $\left.J_{5^{\prime}, 6^{\prime}}=8,4 \mathrm{~Hz}, 1 \mathrm{H}, 5^{\prime}\right), 7,04\left(\mathrm{~d}, J_{5^{\prime \prime}, 6^{\prime \prime}}=8,6 \mathrm{~Hz}, 1 \mathrm{H}\right.$, 5'”), 7,17 - 7,30 (m, 1H, 6'), 7,43 - 7,51 (m, 1H, 2'), 7,65 - 7,85 (m, 3H, 2", 6" e 6"'), 7,99 - 8,14 (m, 1H, 2"”), 10,62 (s, 1H, 7"), 10,93 (s, 1H, 7'), 11,04 (s, 1H, 7'"'). RMN de ${ }^{13} \mathrm{C}\left(75 \mathrm{MHz}, \mathrm{DMSO}-\mathrm{d}_{6}\right) \delta$ 110,0 (3'), 110,3 (3"'), 115,3 (7), 115,9 (3" e 5"), 116,9 (5"'), 117,2 (5'), 121,1 (1'), 123,3 (5), 126,1 (1'”'), 127,8 (1'”), 130,4 (6'), 132,4 (6”"), 132,7 (2" e 6"), 133,9 (2'), 136,0 (2'”'), 145,8 (4), 155,0 (3), 156,2 (4'), 156,5 (4"'), 163,7 (4”), 166,2 (2), 188,0 (6). EM, m/z (\%) $556\left(\left[\mathrm{M}^{+}\right], \mathrm{C}_{24} \mathrm{H}_{14} \mathrm{Br}_{2} \mathrm{O}_{6}, 9\right), 557\left([\mathrm{M}+1]^{+}, 3\right), 558\left([\mathrm{M}+2]^{+}, 17\right)$, $559\left([\mathrm{M}+3]^{+}, 5\right), 560\left([\mathrm{M}+4]^{+}, 9\right), 480(10), 479(5), 478(10), 437$ (9), 214 (10), 212 (10), 134 (12), 122 (8), 121 (100), 113 (6), 105 (12), 93 (19), 82(7), 80 (7), 70 (7), 65 (11). HRMS [ESI(+), IT-TOF]: calculado $\left[\mathrm{C}_{24} \mathrm{H}_{15} \mathrm{Br}_{2} \mathrm{O}_{6}\right]^{+}$556,9230; encontrado 556,9229.

(Z)-4-(3-bromo-4-hidroxifenil)-5-(3,5-dibromo-4-hidroxi-

benzilideno)-3-(4-hidroxibenzoil)furan-2(5H)-ona (16h)

O composto $\mathbf{1 6 h}$ foi obtido como um sólido marrom, em $41 \%$ de rendimento, após purificação por cromatografia em coluna de sílica gel. $\mathrm{T}_{\mathrm{f}}=158,5-159,2{ }^{\circ} \mathrm{C} . \mathrm{R}_{\mathrm{f}}=0,28$ (hexano: acetato de etila, $1: 3 \mathrm{v} / \mathrm{v}) . \mathrm{IV}\left(\mathrm{cm}^{-1}\right) \bar{v}_{\text {max }}: 3307,2921,2852,2162,1980,1743,1643$, 1566, 1474, 1372, 1291, 1158, 983, 869, 808, 610, 424. RMN de ${ }^{1} \mathrm{H}\left(300 \mathrm{MHz}, \mathrm{DMSO}-\mathrm{d}_{6}\right) \delta 6,39(\mathrm{~s}, 1 \mathrm{H}, 7), 6,77$ (d, $J_{3^{\prime \prime}, 2^{\prime,}}=J_{5, ", 6},=$ $8,5 \mathrm{~Hz}, 2 \mathrm{H}, 3$ '"' e 5'”), 6,97 (d, $J_{5,6}=8,4 \mathrm{~Hz}, 1 \mathrm{H}, 5^{\prime}$ ), 7,18 - 7,31 (m, 1H, 6'), 7,41 - 7,57 (m, 1H, 2'), 7,72 (d, $J_{2 ", 3^{\prime \prime}}=J_{6 ",}{ }^{\prime \prime}=8,5 \mathrm{~Hz}$, 2H, 2" e 6"), 8,11 (s, 2H, 2"' e 6"'), 10,64 (s, 1H, 7"), 10,95 (s, 1H, $\left.7^{\prime}\right)$. RMN de ${ }^{13} \mathrm{C}\left(75 \mathrm{MHz}, \mathrm{DMSO}-\mathrm{d}_{6}\right) \delta 110,1$ (5'), 112,4 (7), 113,4
(3'), 116,0 (3" e 5"), 116,9 (1"), 120,9 (3"” e 5"'), 123,9 (5), 127,7 (6'), 127,9 (1'), 130,5 (1'”), 132,8 (2"' e 6"), 134,0 (2'), 135,0 (2"'” e 6"'), 146,7 (4), 152,4 (3), 154,8 (4"”), 156,6 (4'), 163,8 (4"'), 166,0 (2), 187,9 (6). EM, $m / z(\%) 634\left(\left[\mathrm{M}^{+}\right], \mathrm{C}_{24} \mathrm{H}_{13} \mathrm{Br}_{3} \mathrm{O}_{6}, 3\right), 636\left([\mathrm{M}+2]^{+}\right.$, 9), $638\left([\mathrm{M}+4]^{+}, 9\right), 640\left([\mathrm{M}+6]^{+}, 3\right), 560(6), 558(13), 556(6), 214$ (5), 212 (5), 122 (8), 121 (100), 105 (7), 93 (19), 82 (7), 80 (6), 65 (10). HRMS [ESI(+), IT-TOF]: calculado $\left[\mathrm{C}_{24} \mathrm{H}_{13} \mathrm{Br}_{3} \mathrm{O}_{6}\right]^{+} 634,8335$; encontrado 634,8340 .

(Z)-4-(3-bromo-4-hidroxifenil)-5-(3,5-dibromo-4-hidroxibenzilideno)-3-(4-metoxibenzoil)furan-2(5H)-ona (16h')

O composto 16h' foi obtido como um sólido laranja, em $25 \%$ de rendimento, após purificação por cromatografia em coluna de sílica gel. $\mathrm{T}_{\mathrm{f}}=106,1-106,8^{\circ} \mathrm{C} \cdot \mathrm{R}_{\mathrm{f}}=0,36$ (hexano: acetato de etila, $1: 3 \mathrm{v} / \mathrm{v}$ ). IV $\left(\mathrm{cm}^{-1}\right) \bar{v}_{\max }: 2921,2851,2163,2035,1750,1648,1596,1477,1362$, $1295,1254,1162,1021,975,868,794,699,608,520$. RMN de ${ }^{1} \mathrm{H}$ $\left(300 \mathrm{MHz}, \mathrm{DMSO}_{6}\right) \delta 3,81$ (s, 3H, 7"'), 6,40 (s, 1H, 7), 6,97 (d, $J_{5^{\prime}, 6^{\prime}}=J_{3^{\prime \prime}, 2^{\prime \prime}}=J_{5^{\prime \prime}, 6^{\prime \prime}}=8,6 \mathrm{~Hz}, 3 \mathrm{H}, 5^{\prime}, 3^{\prime \prime}$ e 5"), 7,14- 7,35 (m, $1 \mathrm{H}$, 6'), 7,37- 7,54 (m, 1H, 2'), 7,84 (d, $J_{2,3} 3^{\prime \prime}=J_{6 ", 5}=8,6 \mathrm{~Hz}, 2 \mathrm{H}, 2$ "' e 6"), 8,12 (s, 2H, 2"' e 6"'), 10,95 (s, 1H, 7'). RMN de ${ }^{13} \mathrm{C}$ (75 MHz, DMSO-d $_{6}$ ) $\delta 56,2$ (7”), 110,1 (5), 112,4 (7), 113,6 (3'), 114,6 (3"' e 5"), 116,9 (1"), 120,8 (3"' e 5"'), 123,7 (5), 127,9 (1'), 129,0 (6'), 130,5 (1"'), 132,5 (2" e 6"), 134,0 (2'), 135,0 (2'” e 6"'), 146,8 (4), 152,4 (3), 155,3 (4'”), 156,7 (4'), 164,6 (4”), 166,0 (2), 188,2 (6). EM, $m / z$ (\%) $648\left(\left[\mathrm{M}^{+}\right], \mathrm{C}_{25} \mathrm{H}_{15} \mathrm{Br}_{3} \mathrm{O}_{6}, 4\right), 649$ ([M+1] $\left.]^{+}, 2\right), 650$ $\left([\mathrm{M}+2]^{+}, 12\right), 651\left([\mathrm{M}+3]^{+}, 4\right), 652\left([\mathrm{M}+4]^{+}, 12\right), 653\left([\mathrm{M}+5]^{+}, 4\right), 654$ $\left([\mathrm{M}+6]^{+}, 4\right), 573$ (5), 572 (13), 570 (7), 136 (9), 135 (100), 107 (15), 92 (9), 77 (17). HRMS [ESI(+), IT-TOF]: calculado $\left[\mathrm{C}_{25} \mathrm{H}_{16} \mathrm{Br}_{3} \mathrm{O}_{6}\right]^{+}$ 648,8992 ; encontrado 648,8999.

\section{(Z)-4-(3-bromo-4-hidroxifenil)-5-(2,5-dihidroxibenzilideno)- \\ 3-(4-hidroxibenzoil) furan-2(5H)-ona (16i)}

O composto 16i foi obtido como um sólido vermelho escuro, em $78 \%$ de rendimento, após purificação por cromatografia em coluna de sílica gel. $\mathrm{T}_{\mathrm{f}}=148,3-149,1^{\circ} \mathrm{C} . \mathrm{R}_{\mathrm{f}}=0,34$ (hexano: acetato de etila, $1: 3 \mathrm{v} / \mathrm{v})$. IV $\left(\mathrm{cm}^{-1}\right) \bar{v}_{\max }: 3627,3282,3061,2926,2167,2011$, 1710, 1631, 1560, 1503, 1454, 1352, 1229, 1166,1069, 995, 873, $746,647,579,435$. RMN de ${ }^{1} \mathrm{H}\left(300 \mathrm{MHz}\right.$, Acetona-d $\left.{ }_{6}\right) \delta 6,74-$ 6,93 (m, 5H, 7, 3", 5', 3"' e 4'”), 7,08 (d, $J_{5,6}=8,4 \mathrm{~Hz}, 1 \mathrm{H}, 5^{\prime}$ ), 7,35 (dd, $J_{6^{\prime}, 5},=8,4 \mathrm{~Hz}$ e $J_{6^{\prime}, 2^{\prime}}=1,6 \mathrm{~Hz}, 1 \mathrm{H}, 6^{\prime}$ ), 7,68-7,72 (m, 2H, 2' e 6"'), 7,85 (d, $J_{2 ", 3 "}=J_{6 ", 5}=8,6 \mathrm{~Hz}, 2 \mathrm{H}, 2$ "' e 6"), 8,21 (s, $1 \mathrm{H}$, 8 "'), 8,60 (s, 1H, 8"”), 9,51 (s, 1H, 7"). RMN de ${ }^{13} \mathrm{C}(75 \mathrm{MHz}$, Acetona-d ${ }_{6}$ ) $\delta$ 109,7 (7), 109,9 (3"'), 115,4 (3"' e 5"), 116,0 (3'), 116,4 (5'), 116,5 (6"'), 119,0 (4"”), 120,6 (1'), 122,1 (1"'), 124,0 (5), 128,6 (1'”'), 130,1 (6'), 132,3 (2” e 6"), 133,9 (2'), 146,6 (4), 149,9 (2'”), 150,7 (5'”), 154,7 (3), 155,9 (4'), 162,9 (4”), 165,9 (2), 187,7 (6). EM, $m / z(\%) 494\left(\left[\mathrm{M}^{+}\right], \mathrm{C}_{24} \mathrm{H}_{15} \mathrm{BrO}_{7}, 2.09\right), 496\left([\mathrm{M}+2]^{+}\right.$, 2.06), 453 (5), 452 (19), 451 (8), 450 (19), 408 (7), 406 (7), 372 (14), 371 (7), 342 (5), 328 (5), 279 (8), 251 (6), 250 (8), 226 (5), 223 (6), 185 (5), 136 (6), 122 (11), 121 (100), 93 (24), 82 (9), 80 (9), 65 (17). HRMS [ESI(+), IT-TOF]: calculado $\left[\mathrm{C}_{24} \mathrm{H}_{16} \mathrm{BrO}_{7}\right]^{+}$ 495,0074; encontrado 495,0080.

\section{Ensaios Biológicos}

Para a avaliação das atividades biológicas das lactonas 14a-d e dos análogos aos cadiolídeos 15a-k e 16a-i, utilizou-se o método da microdiluição em placa de poliestireno de 96 poços. A metodologia empregada foi adaptada do documento M27-A2 do Clinical and Laboratory Standards Institute. ${ }^{28}$ Os bioensaios foram realizados com os microrganismos Candida albicans (ATCC18804), Staphylococcus aureus (ATCC29212), Bacillus cereus (ATCC11778) Escherichia coli (ATCC25922) e Salmonella typhimurium (ATCC14028), 
disponíveis na coleção de cultura de microrganismos do Laboratório de Biotecnologia e Bioensaios do Instituto de Ciências Exatas da Universidade Federal de Minas Gerais (ICEx-UFMG).

Os microrganismos foram incubados em estufa a $37^{\circ} \mathrm{C}$ por $36 \mathrm{~h}$ e posteriormente suspendidos em água destilada estéril em tubos de ensaio. Após homogeneização, a concentração das suspensões foi ajustada para se obter inóculos contendo $10^{6}$ células $\mathrm{mL}^{-1}$.

Cada composto (14a-d, 15a-k e 16a-i) foi dissolvido em dimetilsulfóxido (DMSO) na concentração de $12,5 \mathrm{mg} \mathrm{mL}^{-1}$. Alíquota de $40 \mu \mathrm{L}$ dessa solução foram adicionadas à $960 \mu \mathrm{L}$ do meio de cultura utilizado no bioensaio (BHI para bactérias e Sabouraud para a levedura), obtendo uma solução de trabalho na concentração de $500 \mu \mathrm{g} \mathrm{mL}^{-1}$ de cada composto e com porcentagem de $4 \%$ (v/v) de DMSO. Foram feitas diluições seriadas de modo que as concentrações testadas dos compostos foram 250,00, 125,00, 62,50, 31,25, 15,63, $7,81,3,90$ e $1,95 \mu \mathrm{g} \mathrm{mL} \mathrm{m}^{-1}$.

Os seguintes experimentos-controle foram realizados conforme descrito por Borel et al.: ${ }^{29}$ (i) controle de crescimento dos microrganismos, visando avaliar a viabilidade celular; (ii) branco, solução sem o inóculo do microrganismo, com finalidade de eliminar o efeito da coloração da solução das substâncias teste; (iii) controle positivo, no qual as substâncias teste foram substituídas pelo antifúngico Miconazol ou pelo antibiótico Ampicilina; (iv) controle do grau de esterilidade do meio de cultura, contendo $100 \mu \mathrm{L}$ de meio de cultura e $100 \mu \mathrm{L}$ de água destilada.

Após incubação das microplacas por 24 horas a $37^{\circ} \mathrm{C}$, o crescimento dos microrganismos foi quantificado pela determinação da densidade óptica por meio da absorbância a $490 \mathrm{~nm}$, utilizando um leitor de microplacas. Inicialmente foi avaliada a inibição de crescimento causada por todos os compostos na concentração de $250 \mu \mathrm{g} \mathrm{mL}^{-1}$. Para as amostras que causaram inibição superior a $50 \%$ na concentração mais elevada foram determinados os valores de $\mathrm{IC}_{50}$. Todos os experimentos foram realizados em duplicata, e os resultados registrados como a média \pm desvio-padrão.

\section{RESULTADOS E DISCUSSÃO}

\section{Síntese}

A estratégia escolhida para a síntese dos análogos aos cadiolídeos baseia-se na metodologia desenvolvida por Boukouvalas e Thibault, ${ }^{18}$ que envolve a reação entre uma inona e o 5-etoxi-4-metiloxazol (6) (Esquema 1, Estratégia 3). O oxazol 6 é disponível comercialmente.
Porém, devido ao elevado custo, foi preparado a partir da $d$, $l$-alanina, conforme procedimento descrito por Dean et al.. ${ }^{30}$

Para a síntese das inonas foram utilizadas duas metodologias, conforme sumarizado no Esquema 2.

A primeira estratégia investigada envolveu a reação entre um acetileto de lítio e um aldeído, seguido por oxidação do álcool resultante (Esquema 2). Essa metodologia consistiu na reação entre o benzaldeído 7a com trifenilfosfina e tetracloreto de carbono, resultando na formação do (2,2-dibromovinil)benzeno como intermediário. ${ }^{31}$ $\mathrm{O}$ tratamento desse intermediário com LDA gerou o composto $\mathbf{8 a}$ (não isolado), que reagido com $p$-metoxibenzaldeído gerou o álcool propargílico correspondente. A oxidação desse álcool, sem purificação prévia, com dióxido de manganês resultou na cetona 11a com $86 \%$ de rendimento. A unidade inona de 11a foi identificada no espectro de $\mathrm{RMN}$ de ${ }^{13} \mathrm{C}$ em 86,8, 93,5 e 176,8 ppm, correspondentes aos carbonos do grupo $\mathrm{C} \equiv \mathrm{C}$ e $\mathrm{C}=\mathrm{O}$. No espectro no infravermelho foram observadas bandas de absorção em $2188 \mathrm{~cm}^{-1} \mathrm{e} 1629 \mathrm{~cm}^{-1}$ associadas aos estiramentos das ligações $\mathrm{C} \equiv \mathrm{C}$ e $\mathrm{C}=\mathrm{O}$, respectivamente. ${ }^{32}$

Para a obtenção do composto $\mathbf{1 1 b}$ por essa metodologia, foi avaliada a reação do aldeído bromado $\mathbf{7 b}$ nas mesmas condições empregadas para o aldeído 7a. Entretanto, nesse caso, verificou-se a remoção do átomo de bromo do anel aromático com formação do mesmo intermediário 8a. Embora a reação de transmetalação de brometos de arila seja comumente realizada com BuLi, ${ }^{33}$ não é incomum que ela também ocorra com o uso de LDA.

Em função do resultado indesejado obtido anteriormente, os alquinos halogenados foram então preparados por meio de uma reação de acoplamento de Sonogashira entre o alquino 9 e cloretos de ácidos carboxílicos 10a-c, empregando-se catalisadores de paládio $^{34}$ (Esquema 2). O emprego dessa metodologia resultou na obtenção dos compostos 11b-d em rendimentos altos (78-97\%). O maior rendimento foi obtido quando utilizado o cloreto de ácido $\mathbf{1 0 b}$, enquanto o emprego do cloreto de $p$-metoxibenzoíla (10a) resultou no menor rendimento (78\%). Esses resultados podem ser entendidos ao se analisar o ciclo catalítico da reação de Sonogashira. Na etapa da adição oxidativa, um complexo de paládio (0), rico em elétrons, reage com o haleto de arila gerando a espécie de paládio (II). Assim, quanto mais eletrofílico for o haleto de arila, mais favorável torna-se esta etapa, em consonância com os resultados obtidos. ${ }^{34}$ Os dados espectroscópicos dos compostos 11b-d são similares aos obtidos para o composto 11a e estão todos em conformidade com o esperado.

Uma vez obtidas as inonas 11a-d, estas foram submetidos à reação Diels-Alder/retro-Diels-Alder seguida de hidrólise para formação das<smiles>[R]c1cc(C(=O)C#CC)ccc1OC</smiles><smiles>[R]c1cc(C#CI)ccc1OC</smiles>

iii, iv

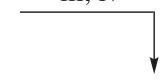<smiles>C#Cc1ccc(OC)c(Br)c1</smiles>

9<smiles>[R]c1cc(C(=O)Cl)cc([R])c1[R]</smiles><smiles>[R]c1cc(C#CC(=O)c2cc([R2])c([R3])c([R])c2)ccc1OC</smiles>

V 11a $\mathrm{R}_{1}=\mathrm{R}_{2}=\mathrm{H} ; \mathrm{R}_{3}=\mathrm{OCH}_{3}(86 \%)$
11b $\mathrm{R}_{1}=\mathrm{Br} ; \mathrm{R}_{2}=\mathrm{H} ; \mathrm{R}_{3}=\mathrm{OCH}_{3}(78 \%)$
11c $\mathrm{R}_{1}=\mathrm{Br} ; \mathrm{R}_{2}=\mathrm{R}_{3}=\mathrm{H}(97 \%)$
11d $\mathrm{R}_{1}=\mathrm{R}_{2}=\mathrm{Br} ; \mathrm{R}_{3}=\mathrm{OCH}_{3}(85 \%)$

10a $\mathrm{R}_{2}=\mathrm{H} ; \mathrm{R}_{3}=\mathrm{OCH}_{3}$

$10 \mathrm{~b} \mathrm{R}_{2}=\mathrm{R}_{3}=\mathrm{H}$

10c $\mathrm{R}_{2}=\mathrm{Br} ; \mathrm{R}_{3}=\mathrm{OCH}_{3}$

Esquema 2. Síntese das inonas 11a-d. Reagentes e condições (i) $\mathrm{PPh}_{3}, \mathrm{CBr}_{4}, \mathrm{CH}_{2} \mathrm{Cl}_{2}, \mathrm{O}^{\circ} \mathrm{C}$ para $25^{\circ} \mathrm{C}, 4 \mathrm{~h}$; (ii) LDA, - $78^{\circ} \mathrm{C}, 1 \mathrm{~h}$; (iii) p-metoxibenzaldeído, $\mathrm{THF},-78{ }^{\circ} \mathrm{C}$ para $25^{\circ} \mathrm{C}, 3 \mathrm{~h}$; (iv) $\mathrm{MnO}_{2}, \mathrm{CH}_{2} \mathrm{Cl}_{2}, 25^{\circ} \mathrm{C}, 17 \mathrm{~h}$; (v) $\mathrm{Pd}\left(\mathrm{PPh}_{3}\right)_{2} \mathrm{Cl}_{2}, \mathrm{CuI}, \mathrm{Et} \mathrm{t}_{3} \mathrm{~N}, \mathrm{THF}, 25^{\circ} \mathrm{C}, 3 \mathrm{~h}$. 
lactonas 14a-d ${ }^{18}$ (Esquema 3). Nesta reação formam-se adutos de Diels-Alder (12a-d) que, nas condições reacionais, sofrem uma cicloreversão com perda de uma molécula de acetonitrila e consequente formação dos intermediários furânicos 13a-d. Esses intermediários foram submetidos à reação de hidrólise com $\mathrm{HBr}$ sem purificação prévia e forneceram as lactonas 14a-d com $81 \%, 51 \%, 79 \%$ e $61 \%$ de rendimento, respectivamente.

As lactonas foram caracterizadas por meio de análises espectroscópicas, destacando-se os seguintes dados: no espectro no infravermelho foram observadas bandas de absorção em torno de 1650 e 1740 $\mathrm{cm}^{-1}$ associadas aos estiramentos das ligações $\mathrm{C}=\mathrm{O}$ das carbonilas da cetona e da lactona, respectivamente. Os espectros de RMN de ${ }^{13} \mathrm{C}$ apresentam sinais em 171,0 e 190,0 ppm atribuídos aos átomos de carbono carbonílicos.

As lactonas intermediárias 14a-d foram submetidas à reação de condensação de Knoevenagel viníloga com benzaldeídos substituídos, disponíveis comercialmente, visando a instalação do grupo benzilideno na posição gama (Esquema 4). Inicialmente, a lactona $\mathbf{1 4 b}$ foi submetida às condições relatadas para a síntese dos cadiolídeos $\mathrm{A}, \mathrm{B}$ e $\mathrm{D}^{18}$, que consistiram na reação com $p$-metoxibenzaldeído em metanol na presença de piperidina. Embora Boukouvalas e Thibault ${ }^{18}$ tenham obtido os alquilidenos com rendimentos de 65 $80 \%$, o produto esperado (15b) foi isolado em apenas 30\%. Essa reação foi realizada várias vezes com pequenas alterações nas condições reacionais (temperatura, tempo, equivalentes molares da base) e não ocorreu melhorias de rendimento reacional. Em função desses resultados, as lactonas foram submetidas à reação de aquilidenação nas condições desenvolvidas por Boukouvalas et al..$^{35}$ e anteriormente empregadas com sucesso para o preparo de vários análogos de nostoclídeos ${ }^{36-39}$ e rubrolídeos. ${ }^{19-24}$ Para isso, as lactonas foram submetidas à reação com os aldeídos na presença de TBDMSOTf e DIPEA. Esse procedimento resulta na formação de um sililfurano intermediário que condensa com o aldeído resultando na formação de um sililéter intermediário. Posterior adição de DBU à mistura reacional resulta na eliminação do grupo -OTBDMS e formação estereosseletiva do alquilideno com configuração $Z$. Empregando essa metodologia, os produtos 15a-k foram obtidos em rendimentos na faixa de 45-89\% (Esquema 4). Os menores rendimentos foram obtidos quando empregado o 3,5-dibromo-4-metoxibenzaldeido. A baixa reatividade desse aldeído já havia sido relatada durante a tentativa de síntese de rubrolídeos naturais. ${ }^{40-42}$

Finalmente, o tratamento dos compostos $\mathbf{1 5 a} \mathbf{a}-\mathbf{k}$ com $\mathrm{BBr}_{3}$ resultou na obtenção dos derivados desmetilados 16a-i. Os rendimentos das reações de desmetilação dos análogos metoxilados 15a-c, 15e-g e $\mathbf{1 5 i}$ variaram de $64-86 \%$. Quando foram utilizados os análogos metoxilados $\mathbf{1 5 d}$ e $\mathbf{1 5 h}$, os rendimentos foram menores (25-45\%) sendo que, para a reação de desmetilação de $\mathbf{1 5 h}$, foi possível a obtenção do composto completamente desmetilado com $41 \%$ de rendimento, 16h, e o composto 16h', com duas hidroxilas e uma metoxila, com $25 \%$ de rendimento. Já na desmetilação dos análogos $\mathbf{1 5} \mathbf{j}$ e $\mathbf{1 5 k}$, não foi possível a obtenção dos produtos hidroxilados. Essa reação foi realizada várias vezes com pequenas alterações nas condições reacionais (temperatura, variação dos equivalentes molares de $\mathrm{BBr}_{3}$, tempo reacional) e não ocorreu melhorias de rendimentos. Desta forma, foi observado que a presença de mais de um átomo bromo em um mesmo anel aromático dificulta a ocorrência da desmetilação
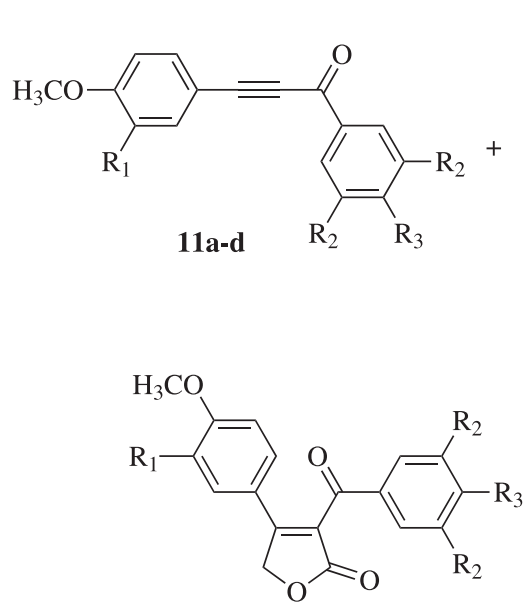

14a-d

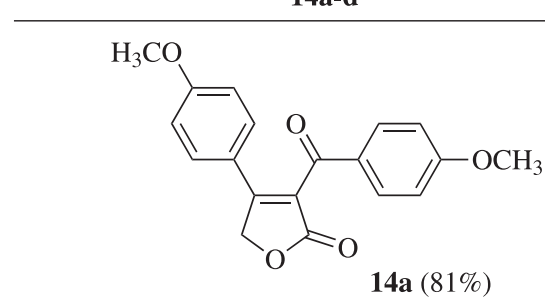

$14 \mathbf{a}(81 \%)$

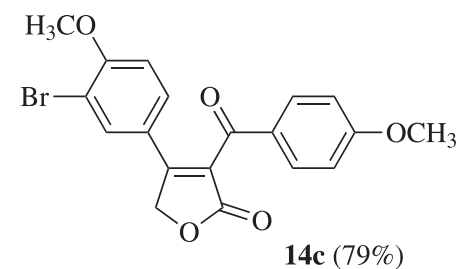

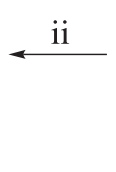
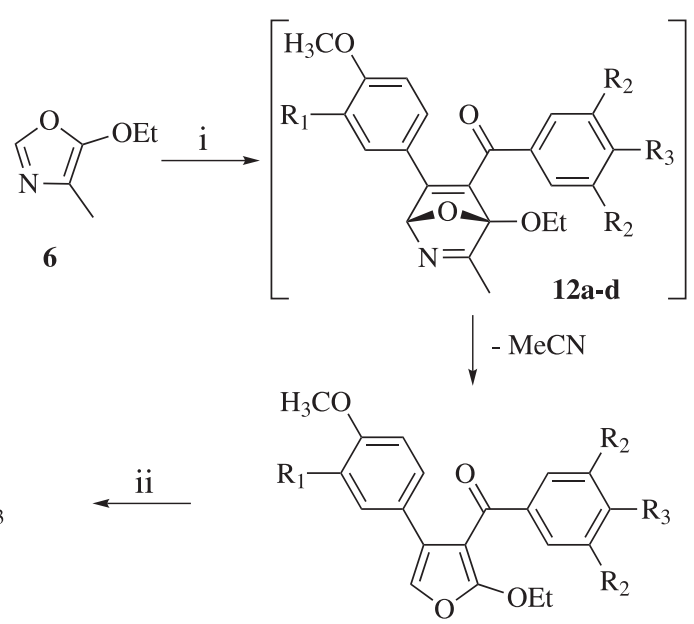

13a-d (não purificado)

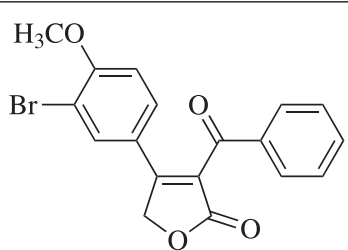

$14 \mathbf{b}(51 \%)$

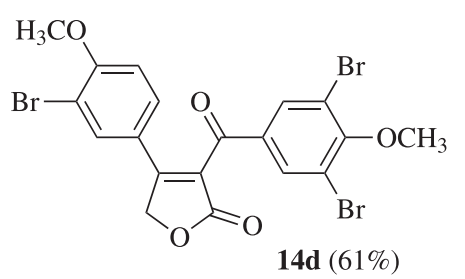

Esquema 3. Síntese das lactonas 14a-d. Reagentes e condições (i) xileno, $150^{\circ} \mathrm{C}, 24 \mathrm{~h}$; (ii) $\mathrm{HBr}(\mathrm{aq}),. \mathrm{THF}, 25^{\circ} \mathrm{C}, 8 \mathrm{~h}$. 


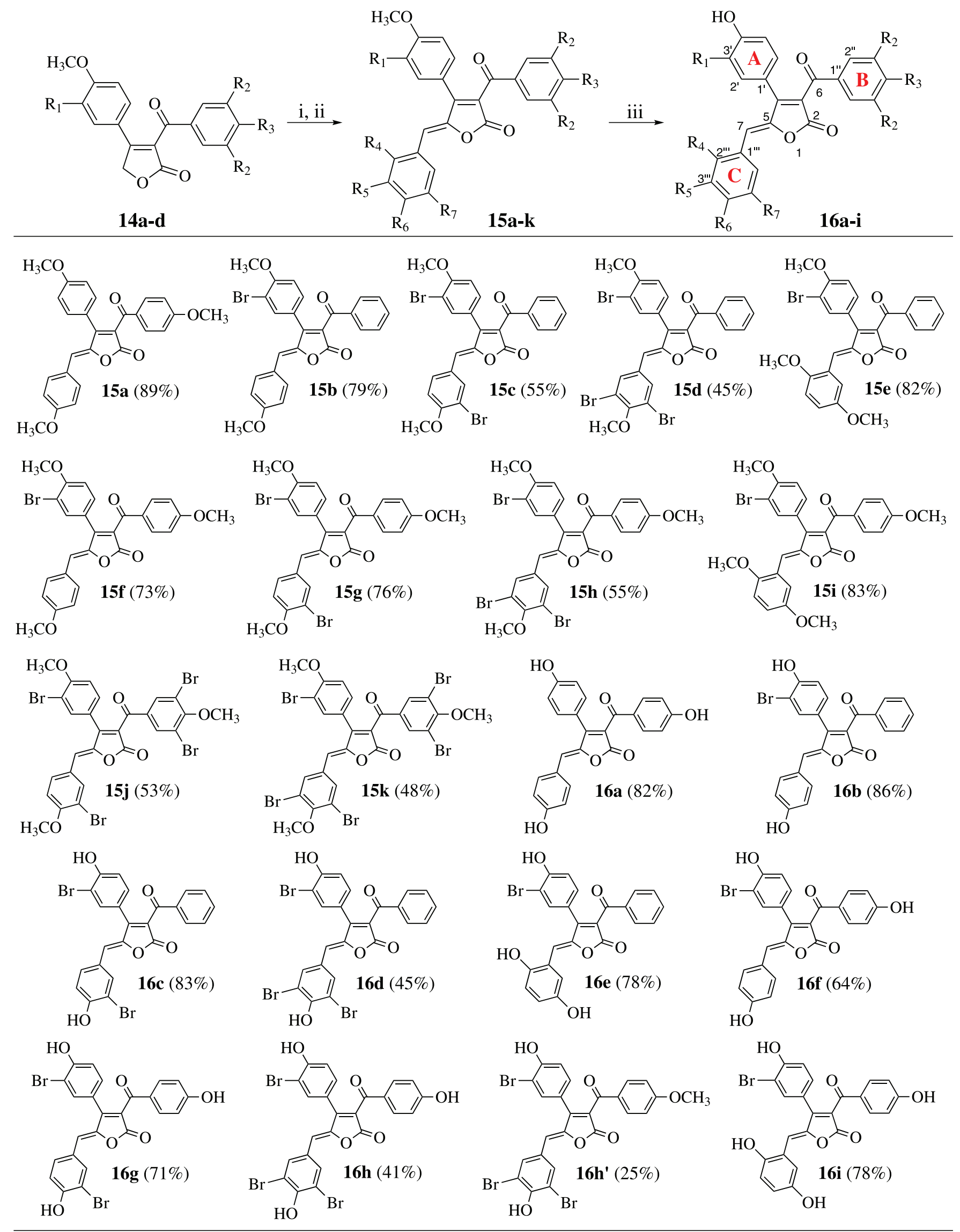

Esquema 4. Síntese dos análogos aos cadiolídeos 16-36. Reagentes e condições: (i) Aldeído, TBDMSOTf, DIPEA, $\mathrm{CH}_{2} \mathrm{Cl}_{2}, 25^{\circ} \mathrm{C}, 2 \mathrm{~h}$; (ii) DBU, refluxo, $2 \mathrm{~h}$; (iii) $\mathrm{BBr}_{3}, \mathrm{CH}_{2} \mathrm{Cl}_{2},-78^{\circ} \mathrm{C}$ para $25^{\circ} \mathrm{C}, 20 \mathrm{~h}$. 
por $\mathrm{BBr}_{3}$, ocasionando rendimentos baixos e em algumas situações a não ocorrência da reação.

Todos os compostos tiveram suas estruturas elucidadas pelas análises dos espectros no infravermelho, RMN de ${ }^{1} \mathrm{H}$ e de ${ }^{13} \mathrm{C}$, e de massas. Experimentos bidimensionais de RMN (COSY, NOESY, $H S Q C$ e $H M B C$ ) também foram realizados em alguns casos para fins de atribuição dos sinais de ${ }^{13} \mathrm{C}$ e de ${ }^{1} \mathrm{H}$ (ver Material Suplementar para todos os espectros de RMN). No geral, para todos os análogos aos cadiolídeos, observou-se o sinal do hidrogênio H-7 como um simpleto em aproximadamente $\delta=6,12$ para os análogos metoxilados e em $\delta=6,35$ para os hidroxilados. Foram observados sinais das metoxilas em torno de $\delta=3,79-3,96$. As posições e multiplicidades dos sinais dos demais hidrogênios variaram em função do padrão de substituição dos anéis aromáticos e as atribuições foram confirmadas com bases nas correlações dos mapas de contorno COSY e NOESY (ver figuras no Material Suplementar). Para as atribuições dos sinais dos espectros de $\mathrm{RMN}$ de ${ }^{13} \mathrm{C}$ foi fundamental a análise dos mapas de contorno $H S Q C$ e $H M B C$, uma vez que foram observados diversos sinais com valores de deslocamentos químicos muito próximos. Por exemplo, no caso do composto 15a, os sinais de C-4', C-4"' e C-4", foram observados em $\delta=161,3,164,2$ e 161,0, respectivamente. As correlações com os sinais em $\delta=3,80$ (H-7'), $\delta=3,82$ (H-7"') e $\delta=3,85$ (H-7"') permitiram as atribuições inequívocas dos mesmos (Figura 36S, Material Suplementar).

\section{Avaliação da atividade antimicrobiana}

Os compostos 14a-d, 15a-k e 16a-i foram submetidos a ensaios in vitro para avaliação de seus efeitos inibitórios sobre o crescimento de S. aureus, E. coli, S. typhimurium e B. cereus, bactérias oportunistas de relevância clínica por acometer pacientes pós-cirúrgicos e imunocomprometidos. ${ }^{43-46}$ Também foram avaliadas as atividades dos compostos contra C. albicans, fungo patogênico frequentemente associado às infecções relatadas em unidades hematológicas e de tratamento intensivo. ${ }^{47}$ Inicialmente foi feita uma avaliação das atividades dos compostos na concentração de $250 \mu \mathrm{g} \mathrm{mL}-1$, conforme resultados apresentados na Tabela 1 .

Os produtos comerciais Ampicilina e Miconazol foram utilizados como controles-positivos, na mesma concentração dos compostos avaliados. Pelos dados obtidos (Tabela 1), verificou-se que todos os compostos avaliados causaram mais de $50 \%$ de inibição em pelo menos uma espécie de microrganismo. Esses resultados indicam claramente que o padrão e a natureza dos grupos substituintes nos análogos aos cadiolídeos são importantes para as atividades. Além disso, as atividades das lactonas $\mathbf{1 4 a - d}$ sugerem o anel butenolídeo é essencial para a atividade biocida, mesmo sem a presença do grupo benzilideno.

O efeito de substituintes nos anéis aromáticos foi avaliado comparando-se as atividades dos pares de compostos metoxilados e

Tabela 1. Efeito dos compostos 14a-d, 15a-k e 16a-i sobre o crescimento de microrganismos patogênicos ${ }^{\mathrm{a}}$

\begin{tabular}{|c|c|c|c|c|c|}
\hline \multirow[b]{2}{*}{ Compostos } & \multicolumn{5}{|c|}{ Microrganismos } \\
\hline & $\begin{array}{c}\text { S. aureus } \\
\text { (ATCC29212) }\end{array}$ & $\begin{array}{c}\text { E. coli } \\
\text { (ATCC25922) }\end{array}$ & $\begin{array}{l}\text { S. typhimurium } \\
\text { (ATCC14028) }\end{array}$ & $\begin{array}{c}\text { B. cereus } \\
\text { (ATCC11778) }\end{array}$ & $\begin{array}{c}\text { C. albicans } \\
\text { (ATCC18804) }\end{array}$ \\
\hline $14 a$ & $89,5 \pm 2,8$ & $25,6 \pm 2,1$ & 0,0 & 0,0 & 0,0 \\
\hline $14 b$ & $80,3 \pm 3,3$ & $16,2 \pm 3,4$ & 0,0 & $30,8 \pm 1,6$ & 0,0 \\
\hline $14 c$ & $73,8 \pm 2,4$ & $19,9 \pm 1,2$ & $2,1 \pm 0,5$ & $30,2 \pm 1,3$ & $14,2 \pm 0,5$ \\
\hline 14d & $74,7 \pm 2,5$ & $4,1 \pm 0,6$ & 0,0 & $48,0 \pm 1,4$ & 0,0 \\
\hline $15 a$ & $64,1 \pm 2,6$ & $4,8 \pm 0,9$ & 0,0 & $48,1 \pm 1,3$ & 0,0 \\
\hline $15 b$ & $79,7 \pm 0,3$ & 0,0 & 0,0 & 0,0 & 0,0 \\
\hline $15 \mathrm{c}$ & $85,3 \pm 1,8$ & $19,0 \pm 0,3$ & 0,0 & 0,0 & $4,7 \pm 1,8$ \\
\hline 15d & $83,2 \pm 2,3$ & $9,1 \pm 1,2$ & $2,2 \pm 0,7$ & $72,9 \pm 2,2$ & $37,8 \pm 1,1$ \\
\hline $15 \mathrm{e}$ & $89,0 \pm 0,1$ & $30,2 \pm 2,9$ & 0,0 & $30,3 \pm 2,4$ & 0,0 \\
\hline $15 f$ & $67,2 \pm 2,8$ & $34,9 \pm 2,5$ & 0,0 & $62,7 \pm 1,6$ & $12,5 \pm 2,8$ \\
\hline $15 \mathrm{~g}$ & $76,3 \pm 2,7$ & $19,9 \pm 1,9$ & $6,7 \pm 2,5$ & $44,1 \pm 0,4$ & 0,0 \\
\hline $15 \mathrm{~h}$ & $79,4 \pm 3,0$ & $29,6 \pm 1,1$ & $19,7 \pm 2,0$ & $42,8 \pm 1,9$ & 0,0 \\
\hline $15 \mathrm{i}$ & $99,9 \pm 0,1$ & $27,2 \pm 2,6$ & $36,6 \pm 2,7$ & $57,5 \pm 1,6$ & $45,8 \pm 2,1$ \\
\hline $15 j$ & $84,0 \pm 0,1$ & $23,8 \pm 2,2$ & $12,9 \pm 0,9$ & 0,0 & 0,0 \\
\hline $15 k$ & $89,4 \pm 1,9$ & $7,3 \pm 1,9$ & 0,0 & $77,2 \pm 2,9$ & $16,5 \pm 3,0$ \\
\hline $16 a$ & $83,2 \pm 2,3$ & 0,0 & $7,7 \pm 0,9$ & $43,0 \pm 2,8$ & $13,3 \pm 1,2$ \\
\hline $16 \mathrm{~b}$ & $88,6 \pm 2,6$ & 0,0 & $7,5 \pm 6,3$ & 0,0 & $22,8 \pm 1,6$ \\
\hline $16 \mathrm{c}$ & $85,7 \pm 2,4$ & 0,0 & $15,3 \pm 2,6$ & 0,0 & 0,0 \\
\hline 16d & $95,9 \pm 2,0$ & $61,4 \pm 1,1$ & $30,9 \pm 1,5$ & $84,2 \pm 1,9$ & 0,0 \\
\hline $16 e$ & $77,4 \pm 2,9$ & $33,1 \pm 2,1$ & 0,0 & 0,0 & $27,9 \pm 3,4$ \\
\hline $16 f$ & $56,9 \pm 2,6$ & 0,0 & $9.6 \pm 1,1$ & $68,5 \pm 1,0$ & $12,9 \pm 0,4$ \\
\hline $16 \mathrm{~g}$ & $98,9 \pm 2,9$ & 0,0 & $38,0 \pm 2,8$ & $40,3 \pm 3,2$ & $17,2 \pm 2,6$ \\
\hline $16 \mathrm{~h}$ & $88,4 \pm 3,2$ & $77,1 \pm 2,0$ & $44,6 \pm 2,7$ & $38,1 \pm 2,4$ & 0,0 \\
\hline $16 h$ ' & $97,7 \pm 2,3$ & $80,8 \pm 1,9$ & $17,0 \pm 2,8$ & $30,5 \pm 2,3$ & 0,0 \\
\hline $16 \mathbf{i}$ & $94,9 \pm 1,0$ & $4,5 \pm 0,2$ & $8,4 \pm 1,6$ & $52,5 \pm 0,6$ & $29,7 \pm 2,8$ \\
\hline Ampicilina & $97,1 \pm 0,1$ & $95,3 \pm 1,0$ & $92,3 \pm 0,8$ & $92,9 \pm 0,1$ & - \\
\hline Miconazol & - & - & - & - & $91,7 \pm 1,4$ \\
\hline
\end{tabular}

a Todos os compostos foram testados na concentração de $250 \mu \mathrm{g} \mathrm{mL} \mathrm{mL}^{-1}$. Os valores correspondem à porcentagem de inibição ( \pm desvio padrão) do crescimento microbiano. 
hidroxilados $(\mathbf{1 5 a} / \mathbf{1 6 a} ; \mathbf{1 5 b} / \mathbf{1 6 b} ; \mathbf{1 5 c} / \mathbf{1 6 c} ; \mathbf{1 5 d} / \mathbf{1 6 d} ; \mathbf{1 5 e} / \mathbf{1 6 e} ; \mathbf{1 5 f} / \mathbf{1 6 f}$; 15g/16g; 15h/16h-h'; 15i/16i) (Tabela 1). Não foi possível estabelecer correlação direta de efeito da metoxila/hidroxila sobre a atividade, uma vez que ocorreu tanto aumento quanto diminuição da atividade quando se considera uma mesma cepa, mas pôde ser observada uma tendência no aumento da atividade para os análogos hidroxilados.

Outro parâmetro avaliado foi o padrão de substituintes dos três anéis aromáticos, A, B e C. Observou-se um aumento da atividade com a presença do bromo no anel A. Tal efeito é observado ao serem comparados os derivados $\mathbf{1 5 a} / \mathbf{1 5} \mathbf{b}-\mathbf{k}$ e 16a/16b-i, dos quais os compostos 15a e 16a apresentam apenas metoxila/hidroxila como substituinte no anel A e os compostos 15b-k e 16b-i apresentam metoxila/hidroxila e um átomo de bromo como substituintes. Quanto ao anel B, não foi possível estabelecer uma correlação direta do efeito do substituinte sobre a atividade, uma vez que os compostos 15b-e e 15j-k, que não apresentam substituintes e que possuem um grupo metoxila em para e átomos de bromo em meta, respectivamente, foram mais ativos que os compostos $\mathbf{1 5 f - i}$, que apresentam uma metoxila como substituinte na posição para do anel aromático B. Já para o anel C, observa-se que, com o aumento do padrão de substituição, há uma tendência de aumento da atividade, como pode ser notado para $\mathbf{1 5 b} / \mathbf{1 5 c}-\mathbf{e}, \mathbf{1 5 f} / \mathbf{1 5} \mathrm{g}-\mathbf{i}, \mathbf{1 5 j} / \mathbf{1 5 k}$, 16b/16c-e e 16f/16g-i em relação a $S$. aureus. Para as lactonas 14a-d, também não foi possível estabelecer correlação direta entre a atividade biocida e o padrão de substituição dos anéis aromáticos.

Por outro lado, a análise em conjunto de atividade contra os microrganismos mostrou que os compostos avaliados são mais ativos contra as bactérias Gram-positivas ( $S$. aureus e B. cereus) do que contra as Gram-negativas (E. coli e $S$. typhimurium). Além disso os compostos apresentam baixa atividade frente ao fungo $C$. albicans, mostrando certa seletividade contra os microrganismos testados. Os compostos mais ativos e promissores antibacterianos foram $15 \mathbf{i}$ e 16g, que causaram inibição superior à apresentada pela Ampicilina, controle positivo, sobre $S$. aureus. As porcentagens de inibição causadas pelos compostos 16d, 16h' e 16i também podem ser equiparadas àquelas do antibiótico comercial frente a $S$. aureus. Estes resultados se mostraram bastante relevantes, considerando que atualmente a maioria dos casos relatados de infecções nosocomiais tem sido atribuída a $S$. aureus. ${ }^{43}$

Para os compostos que apresentaram inibição superior a $50 \%$ foram determinados os valores de $\mathrm{IC}_{50}$, e comparados com o controle positivo, Ampicilina, conforme apresentado na Tabela 2.

Como pode ser verificado na Tabela 2, não foi possível calcular os valores de $\mathrm{IC}_{50}$ para todos os microrganismos, uma vez que em diversos casos não se observou uma boa correlação entre atividade-concentração. Para a bactéria S. typhimurium e o fungo C. albicans, nenhum dos compostos avaliados inibiu o crescimento em mais de $50 \%$, impossibilitando, dessa forma, a obtenção da concentração inibitória para estes microrganismos.

No caso de B. cereus e $E$. coli, todos os compostos foram menos ativos que o produto comercial. O composto mais ativo para $B$. cereus foi 15f, com $\mathrm{IC}_{50}=3,6 \mu \mathrm{g} \mathrm{mL} \mathrm{L}^{-1}$, correspondendo a 3,3 vezes o valor encontrado para a Ampicilina. Para E. coli, o composto $\mathbf{1 6 h}$ ' foi o mais ativo, com $\mathrm{IC}_{50}=47,0 \mu \mathrm{g} \mathrm{mL} \mathrm{m}^{-1}$, correspondendo a 20,4 vezes o valor encontrado para o controle positivo.

Para S. aureus, foi possível calcular os valores de $\mathrm{IC}_{50}$ para todos os compostos. Para a maioria das substâncias a atividade apresentada foi inferior ao produto comercial, com exceção de $\mathbf{1 5 b}$ $\left(\mathrm{IC}_{50}=1,8 \mu \mathrm{g} \mathrm{mL}^{-1}\right), 1,8$ vezes mais ativo que a Ampicilina e de $\mathbf{1 4 b}$ $\left(\mathrm{IC}_{50}=3,4 \mu \mathrm{g} \mathrm{mL}^{-1}\right)$, que mostrou atividade próxima à apresentada pela Ampicilina $\left(\mathrm{IC}_{50}=3,3 \mu \mathrm{g} \mathrm{mL} \mathrm{L}^{-1}\right)$. Os compostos $15 \mathrm{c}$ e $16 \mathbf{e}$ apresentaram boa atividade quando comparado com os demais compostos avaliados, ambos com $\mathrm{IC}_{50}=6,0 \mu \mathrm{g} \mathrm{mL} \mathrm{m}^{-1}$, sendo 1,8 vezes o valor encontrado para a Ampicilina. No caso dos compostos 15i, 16d, 16g,
Tabela 2. $\mathrm{IC}_{50}\left(\mu \mathrm{g} \mathrm{mL} \mathrm{m}^{-1}\right)$ das lactonas 14a-d, análogos aos cadiolídeos 15a-k e 16a-i e dos controles positivos

\begin{tabular}{|c|c|c|c|}
\hline \multirow[b]{2}{*}{ Compostos } & \multicolumn{3}{|c|}{$\mathrm{IC}_{50}\left(\mu \mathrm{g} \mathrm{mL}^{-1}\right)$} \\
\hline & $\begin{array}{c}\text { S. aureus } \\
\text { (ATCC29212) }\end{array}$ & $\begin{array}{c}\text { B. cereus } \\
\text { (ATCC11778) }\end{array}$ & $\begin{array}{c}\text { E. coli } \\
\text { (ATCC25922) } \\
\end{array}$ \\
\hline$\overline{14 a}$ & 23,8 & $-\mathrm{a}^{\mathrm{a}}$ & - \\
\hline $14 b$ & 3,4 & - & - \\
\hline $14 c$ & 28,1 & - & - \\
\hline 14d & 30,3 & - & - \\
\hline $15 a$ & 39,8 & - & - \\
\hline $15 b$ & 1,8 & - & - \\
\hline $15 \mathrm{c}$ & 6,0 & - & - \\
\hline 15d & 24,8 & 41,6 & - \\
\hline $15 e$ & 36,1 & - & - \\
\hline $15 f$ & 51,7 & 3,6 & - \\
\hline $15 \mathrm{~g}$ & 36,7 & - & - \\
\hline $15 \mathrm{~h}$ & 51,1 & - & - \\
\hline $15 i$ & 86,1 & 31,0 & - \\
\hline $15 \mathrm{j}$ & 14,9 & - & - \\
\hline $15 k$ & 29,7 & 14,2 & - \\
\hline $16 \mathbf{a}$ & 14,0 & - & - \\
\hline $16 \mathrm{~b}$ & 23,8 & - & - \\
\hline $16 \mathrm{c}$ & 34,1 & - & - \\
\hline 16d & 29,2 & 53,8 & 82,8 \\
\hline $16 e$ & 6,0 & - & - \\
\hline $16 f$ & 23,0 & 61,8 & - \\
\hline $16 \mathrm{~g}$ & 81.5 & - & - \\
\hline $16 \mathrm{~h}$ & 92,4 & - & 50,7 \\
\hline $16 h^{\prime}$ & 11,5 & - & 47,0 \\
\hline $16 \mathbf{i}$ & 30,0 & 12,3 & - \\
\hline Ampicilina & 3,3 & 1,1 & 2,3 \\
\hline
\end{tabular}

a: $\mathrm{IC}_{50}$ não foi determinado.

16h' e 16i que, na concentração de $250 \mu \mathrm{g} \mathrm{mL} \mathrm{m}^{-1}$ apresentaram atividade semelhante ao antibiótico comercial, causando 99,9\%, 95,9\%, $98,9 \%, 97,7 \%$ e $94,9 \%$ de inibição, respectivamente, observou-se valores de $\mathrm{IC}_{50}$ muito elevados.

Em termos de seletividade dos compostos em relação aos diversos microrganismos, verificou-se uma tendência de os compostos serem mais ativos para bactérias Gram-positivas, uma vez que foi possível calcular o valor de $\mathrm{IC}_{50}$ para todos os compostos avaliados para $S$. aureus e alguns para B. cereus. Já para as bactérias Gram-negativas, E. coli e $S$. typhimurium, e para o fungo C. albicans, os compostos apresentaram uma baixa atividade, sendo possível a obtenção dos valores de $\mathrm{IC}_{50}$ somente para $\mathbf{1 6 d}, \mathbf{1 6 h}$ e $\mathbf{1 6 h}$ ' em relação a $E$. coli.

Considerando que a diferença estrutural entre os compostos é bastante limitada, não é possível chegar a uma conclusão sobre a relação estrutura-atividade. Entretanto, pelos resultados dos ensaios biológicos para os compostos mais ativos contra S. aureus (lactona 14b e análogos 15b, 15c e 16e) fica evidente que a ausência de substituinte no anel B e a presença de bromo no anel A influenciaram na atividade.

\section{CONCLUSÃO}

No presente trabalho foi utilizada, como etapa-chave da síntese, a reação de Diels-Alder/retro-Diels-Alder, sendo obtidas quatro lactonas precursoras e 21 análogos aos cadiolídeos. As lactonas 14a-d e os análogos aos cadiolídeos 15a-k e 16a-i foram submetidos a bioensaios para avaliar seus efeitos sobre o crescimento microbiano de $S$. 
aureus, E. coli, S. typhimurium, B. cereus e C. albicans. Os resultados demostraram que os compostos possuem certa seletividade, sendo mais ativos contra as bactérias Gram-positivas (S. aureus e B. cereus) do que as Gram-negativas (E. coli e $S$. typhimurium) e apresentam baixa atividade sobre o fungo $C$. albicans. Os compostos mais ativos e promissores antibacterianos foram a furanona $\mathbf{1 4 b}$ e os análogos 15b, 15c e 16e, que apresentaram melhores inibições para $S$. aureus. Os resultados indicaram também que os efeitos dos compostos sobre os microrganismos podem ser modulados pela alteração dos grupos substituintes e suas posições nas três unidades aromáticas estruturais dos cadiolídeos.

\section{MATERIAL SUPLEMENTAR}

Os espectros de RMN de ${ }^{1} \mathrm{H},{ }^{13} \mathrm{C}$ e bidimensionais das substâncias sintetizadas estão disponíveis em http://quimicanova.sbq.org.br, na forma de arquivo PDF, com acesso livre.

\section{AGRADECIMENTOS}

Ao CNPq e a FAPEMIG pelas bolsas concedidas (JAT e LCAB) e pelo apoio financeiro. Aos técnicos Cristiane Isaac Cerceau pela obtenção dos espectros de RMN, José Luiz Pereira pelos espectros de massas e Márcio José Gomes Alvarenga pelos espectros no infravermelho.

\section{REFERÊNCIAS}

1. Bauer, A.; Brönstrup, M.; Nat. Prod. Rep. 2014, 31, 35.

2. Cragg, G. M.; Newmam, D. J.; Biochim Biophys Acta 2013, 1830, 3670.

3. Gammon, K. M.; Nature 2014, 509, S10.

4. Cuevas, C.; Francesch, A.; Nat. Prod. Rep. 2009, 26, 322.

5. Ledford, H.; Nature 2010, 468, 608.

6. Menna, M.; Phytochem. Rev. 2009, 8, 461.

7. Carroll, A. R.; Avery, V. M.; J. Nat. Prod. 2009, 72, 696.

8. Ebada, S. S.; Lin, W. H.; Proksch, P.; Mar. Drugs 2010, 8, 313.

9. Smith, C. J.; Hettich, R. L.; Jompa, J.; Tahir, A.; Buchanan, M. V.; Ireland, C. M.; J. Org. Chem. 1998, 63, 4147.

10. Wang, W.; Kim, H.; Nam, S-J.; Rho, B. J.; Kang, H.; J. Nat. Prod. 2012, 75, 2049.

11. Won, T. H.; Jeon, J-E.; Kim, S-H.; Lee, S-H.; Rho, B. J.; Oh, D-C.; Oh, K-B.; Shin, J.; J. Nat. Prod. 2012, 75, 2055.

12. Smitha, D.; Kumar, M. M. K.; Ramana, H.; Rao, D. V.; Nat. Prod. Res. 2014, 28, 12 .

13. Hede, K.; Nature 2014, 509, S2.

14. Ahn, C-H.; Won, T-H.; Kim, H.; Shin, J.; Oh, K-B.; Bioorg. Med. Chem. Lett. 2013, 23, 4099.

15. Boukouvalas, J.; Pouliot, M.; Synlett 2005, 343.

16. Peixoto, P. A.; Boulangé, A.; Leleu, S.; Franck, X.; Eur. J. Org. Chem. 2013, 16, 3316 .

17. Boulangé, A.; Parraga, J.; Galán, A.; Cabedo, N.; Leleu, S.; Sanz, M. S.; Cortes, D.; Franck, X.; Bioorg. Med. Chem. 2015, 23, 3618.

18. Boukouvalas, J.; Thibault, C.; J. Org. Chem. 2015, 80, 681.

19. Pereira, U. A.; Barbosa, L. C. A.; Maltha, C. R. A.; Demuner, A. J.; Masood, M. A.; Pimenta, A. L.; Bioorg. Med. Chem. Lett. 2014, 24 , 1052.

20. Pereira, U. A.; Barbosa, L. C. A.; Maltha, C. R. A.; Demuner, A. J.; Masood, M. A.; Pimenta, A. L.; Eur. J. Med. Chem. 2014, 82, 127.
21. Varejão, J. O.; Barbosa, L. C.; Varejão, E. V.; Maltha, C. R.; King-Diaz, B.; Hennsen, B. L.; J. Agric. Food Chem. 2014, 62, 5772.

22. Varejão, J. O. S.; Barbosa, L. C. A.; Ramos, G. A.; Varejão, E. V. V.; King-Díaz, B.; Lotina-Hennsen, B.; J. Photochem. Photobiol. B. 2015, $145,11$.

23. Pereira, U. A.; Barbosa, L. C. A.; Demuner, A. J.; Silva, A. A.; Bertazzini, M.; Forlani, G.; Chem. Biodivers. 2015, 12, 987.

24. Pereira, U. A.; Moreira, T. A.; Barbosa, L. C. A.; Maltha, C. R. A.; Bomfim, I. S.; Maranhão, S. S.; Moraes, M. O.; Pessoa, C.; BarrosNepomuceno, F. W. A.; Med. Chem. Comm. 2016, 7, 345.

25. Perrin, D. D.; Armarego, W. L. F.; Purification of Laboratory Chemicals, $3^{\mathrm{a}}$ ed., Pergamon: Oxford, 1988.

26. Casey, M.; Leonard, J.; Lygo, B.; Procter, G. Advanced Practical Organic chemistry, $1^{\text {a }}$ ed., Blackie: Glasgow, 1990.

27. Mairink, S. Z.; Barbosa, L. C. A.; Boukouvalas, J.; Pedroso, S. H. S. P.; Santos, S. G.; Farias, L. M.; Med. Chem. Res. 2018, 27, 2426.

28. CLSI. Clinical and Laboratory Standards Institute. Reference Method for Broth Dilution Antifungal Susceptibility Testing of Yeasts, 3rd Edition M27-A2. v. 22, n. 15, 2002.

29. Borel, C. R.; Barbosa, L. C. A.; Maltha, C. R. A.; Fernandes, S. A.; Santos, L. B.; Takahashi, J. A.; Quím. Nova 2017, 40, 1065.

30. Dean, A.; Ferlin, M. G.; Brun, P.; Castagliuolo, I.; Badocco, D.; Pastore, P.; Venzo, A.; Bombi, G. G.; Di Marco, V. B.; Dalton Trans. 2008, 1689.

31. Rosiak, A.; Frey, W.; Christoffers, J.; Eur. J. Org. Chem. 2006, 4044.

32. Barbosa, L. C. A.; Espectroscopia no Infravermelho na caraterização de compostos orgânicos, $1^{\mathrm{a}}$ ed., Editora UFV: Viçosa, 2011.

33. Parham, W. E.; Bradsher, C. K.; Acc. Chem. Res. 1982, 15, 300.

34. Karak, M.; Barbosa, L. C. A.; Hargaden, G. C.; RSC Adv. 2014, 4, 53442.

35. Boukouvalas, J.; Maltais, F.; Lanchance, N.; Tetrahedron Lett. 1994, 35, 7897.

36. Barbosa, L. C. A.; Varejão. J. O. S.; Petrollino, D.; Pinheiro, P. F.; Demuner, A. J.; Maltha, C. R. A.; Forlani, G.; ARKIVOC 2012, 4, 15.

37. Teixeira, R. R.; Pereira, P. F.; Barbosa, L. C. A.; Carneiro, J. W. M.; Giuseppe F.; Pest. Manag. Sci. 2010, 66, 196.

38. Barbosa, L. C. A.; Maltha, C. R. A.; Demuner, A. J.; Pinheiro, P. F.; Varejão, J. O. S.; Montanari, R. M.; Andrade, N. J.; Quím. Nova 2010, 33, 2020.

39. Teixeira, R. R.; Barbosa, L. C. A.; Carneiro, J. W. M.; Corrêa, R. S.; Ellena, J.; Doriguetto, A. C.; J. Mol. Struct. 2009, 927, 1.

40. Bellina, F.; Anselmi, C.; Rossi, R.; Tetrahedron Lett. 2002, 43, 2023.

41. Boukouvalas, J.; McCann, L. C.; Tetrahedron Lett. 2010, 51, 4636.

42. Karak, M.; Acosta, J. A. M.; Barbosa, L. C. A.; Boukouvalas, J.; Eur. J. Org. Chem. 2016, 3780.

43. Hiramatsu. K.; Katayama, Y.; Matsuo, M.; Sasaki, T.; Morimoto, Y.; Sekiguchi, A.; Baba, T.; J. Infect. Chemother. 2014, $20,593$.

44. Petty, N. K.; Zakour, N. L. B.; Stanton-Cook, M.; Skippington, E.; Totsika, M.; Forde, B. M.; Phan, M.; Moriel, D. G.; Peters, K. M.; Davies, M.; Rogers, B. A.; Dougan, G.; Rodriguez-Baño, J.; Pascual, A.; Pitout, J. D. D.; Upton, M.; Paterson, D. L.; Walsh, T. R.; Schembri, M. A.; Beatson, S. A.; Proc. Nat.l Acad. Sci. USA. 2014, 111, 5694.

45. Miranda, J. L.; Oliveira, M. D. L.; Oliveira, I. S.; Frias, I. A. M.; Franco, O. L.; Andrade, C. A. S.; Biochem. Eng. J. 2017, 124, 108

46. Ikeda, M.; Yagihara, Y.; Tatsuno, K.; Okazaki, M.; Okugawa, S.; Moriya, K.; Ann. Clin. Microbiol. Antimicrob. 2015, 14, 43.

47. Kathiravan, M.; Salake, A. B.; Chothe, A.; Dudhe, P. B.; Watode, R. P.; Mukta, M. S.; Gadwe, S.; Bioorg. Med. Chem. 2012, 20, 5678. 